\title{
A GENERALIZED NOTION OF BOUNDARY $\left({ }^{1}\right)$
}

BY

\section{W. H. FLEMING AND L. C. YOUNG}

This paper reports some recent results in the theory of generalized parametric surfaces. The object of this theory, introduced by Young in [18], is to generalize the elementary notions of parametric surface in such a way that a general principle of minimum holds for surface integral variational problems in parametric form. Since nonregular problems are not excluded, the notion of generalized parametric surface is of necessity more general than the usual concepts of surface, involving in many instances both a track (surface in the ordinary sense, possibly mildly discontinuous) and a linear average over a space of Jacobian matrices at each point of the track (for ordinary surfaces the average is the trivial one in which the normal has weight one and all others weight zero) $\left({ }^{2}\right)$.

In this paper, we first introduce abstract notions of boundary and track, defined for all non-negative linear functionals $L$ on a certain linear space. Since, in particular, every generalized parametric surface is such a linear functional, these definitions apply to all generalized parametric surfaces. We next specialize to admit only boundaries which correspond in a certain way to boundary curves in the sense of classical analysis, and characterize the set of all $L$ with boundary in this class. Finally, we specialize further by imposing a condition of indecomposibility with respect to the boundary.

As an application, some conditions are established for equivalence of indefinite variation problems which have fixed boundary conditions with positive definite problems.

1. Introduction. The main definitions and results of the paper are outlined in this section. Details and auxiliary results are left to later sections.

Let $E^{m}(m \geqq 3)$ denote the space of all continuous functions $f(x, J)$ of an $m$-dimensional vector $x=\left[x^{1}, \cdots, x^{m}\right]$ and an $m \times m$ skew-symmetric matrix $J=\left[j^{r s}\right]$ of rank 2 which satisfy the homogeneity condition $f(x, k J)$ $=k f(x, J)$ for $k \geqq 0$. A mapping $x(u, v)$ from the square $U:[0 \leqq u \leqq 1 ; 0 \leqq v \leqq 1]$ to $m$-space $R^{m}$ is termed a Dirichlet (parametric) representation if:

(1) $x(u, v)$ is absolutely continuous in Tonelli's sense; and

(2) $\iint_{U}\left[x_{u}^{2}+x_{v}^{2}\right] d u d v<\infty$.

Presented to the Society, September 2, 1952; received by the editors February 23, 1953

(1) A number of the results presented here also appear in the first author's Ph.D. Thesis at the University of Wisconsin, August 1951.

(2) The notion of generalized parametric surface is in many respects analogous to that of generalized curve and generalized nonparametric surface introduced a few years ago by Young $[14 ; 15 ; 16]$ (cf. also McShane $[10])$. 
Let $J(u, v)$ denote the matrix of Jacobians $x_{u}^{r} x_{v}^{s}-x_{u}^{s} x_{v}^{r}$ of the partial transformations $x^{r}=x^{r}(u, v), x^{s}=x^{s}(u, v)$ of $x(u, v)$. For convenience we refer to $J(u, v)$ simply as the Jacobian of $x(u, v)$.

Since it is variational problems involving surface integrals which motivate our theory, we begin, as in [18], with a notion of surface expressed directly in terms of these integrals, rather than the more familiar notion of Fréchet surface: A Dirichlet (parametric) surface with Dirichlet representation $x(u, v)$ is the non-negative linear functional $L$ defined, for all $f$ in $E^{m}$, by the surface integral

$$
(L, f)=\iint_{U} f[x(u, v), J(u, v)] d u d v
$$

In this paper the symbol $L$ represents in general an arbitrary non-negative linear functional on $E^{m}$, of which every Dirichlet surface and, more generally, every classical parametric surface is a particular case. As notion of convergence of linear functionals $L$ we use as in [18] $w^{*}$ convergence, i.e., convergence of the numbers $(L, f)$ for all $f$. As used in [18], the notion of generalized parametric surface is equivalent to that of non-negative linear $L$.

We call exact integrands those $\phi$ in $E^{m}$ for which the surface integral $(L, \phi)$ vanishes whenever $L$ is a closed polyhedron. We define the generalized boundary (abbreviated g-boundary) for an arbitrary non-negative linear $L$ to be the linear functional $\lambda(L)$ obtained by restricting the domain of $L$ to exact integrands $\left({ }^{3}\right)$. The $g$-boundary is a generalization of line integration around a boundary curve, paths traversed in opposite senses being allowed to cancel. Similarly, we define the generalized track of $L$ to be the restriction of $L$ to integrands $f$ which are linear in the variable $J$.

A $g$-boundary $\lambda$ is called admissible if $\lambda=\lambda(L)$ for some Dirichlet surface $L$.

(1.1) THEOREM. Let $\lambda_{0}$ be admissible. Then a necessary and sufficieni condition that $L_{0}$ have g-boundary $\lambda\left(L_{0}\right)=\lambda_{0}$ is that $L_{0}$ be the $w^{*}$ limit of a sequence of linear combinations $\sum k_{j} L_{j}$ with positive coefficients $k_{j}$ of Dirichlet surfaces $L_{j}$ with $\lambda\left(L_{j}\right)=\nu_{j} \lambda_{0}$, where each $\nu_{j}$ is a positive integer and $\sum k_{j} \nu_{j}=1$. In the particular case when the dimension $m$ of $x$-space $R^{m}$ is 3 , one can suppose further that all $\nu_{j}=1$.

We term $L$ closed if $\lambda(L)=0 . L \neq 0$ is basic closed if $L=L_{1}+L_{2}$ with $L_{1}, L_{2}$ closed implies $L_{1}=k L$ for some $k, 0 \leqq k \leqq 1$. If $\lambda(L)=\lambda \neq 0, L$ is termed basic ( $\lambda$ ) if $L$ is an extreme point of the set of all functionals with $g$-boundary $\lambda$.

(1.2) Theorem. Suppose $m=3$. Then if $\lambda \neq 0$ is admissible and $L_{0}$ is basic $(\lambda), L_{0}$ is the $w^{*}$ limit of a sequence of Dirichlet surfaces with g-boundary $\lambda$.

In stating the analogue of (1.2) for $\lambda=0$, one must take account of the

$\left({ }^{3}\right)$ In other words, $\lambda(L)$ is defined by the equation $(\lambda(L), \phi)=(L, \phi)$ for all exact $\phi$. 
fact that $k L$ is basic closed whenever $L$ is basic closed and $k>0$ :

(1.3) Theorem. Suppose $m=3$. Then if $L_{0}$ is basic closed, $k L_{0}$ is the $w^{*}$ limit of a sequence of closed Dirichlet surfaces for some $k>0$.

A non-negative linear $L$ is said to be situated in a subset of $A$ of $R^{m}$ if a closed (nonempty) subset $A_{1}$ of $A$ exists such that $(L, f)=0$ whenever $f(x, J)=0$ for all $x$ in $A_{1}$ and all $J . L$ is termed concentrated if $L$ is situated in a. point $x_{0}$.

The methods used to prove (1.1) also yield:

(1.4) Theorem. Let $f_{0}$ be in $E^{m}$ and $A$ a compact, convex subset of $R^{m}$. Then a necessary and sufficient condition that an exact integrand $\phi$ and an $\epsilon>0$ exist such that $f_{0}(x, J) \geqq \phi(x, J)+\epsilon|J|$ for all $x$ in $A$ and all $J$ is that either of the following hold:

(a) $\left(L, f_{0}\right)>0$ for every basic closed $L$ situated in $A$;

(b) There exists an open set $\Omega$ containing $A$ and an $\epsilon_{1}>0$ such that $\left(L, f_{0}\right)$ $\geqq \epsilon_{1}($ area of $L)$ for every closed polyhedron $L$ situated in $\Omega$.

From (1.4) follow conditions for a variational problem with fixed boundary conditions to be equivalent to a positive definite problem. In particular, (1.4) allows the hypothesis of positive definiteness to be weakened in the statement of known theorems $[3 ; 6 ; 13]$ insuring the existence of Dirichlet surface solutions for certain semi-regular problems with fixed boundary conditions (cf. §13).

2. Notation; the norm and modulus. In applying the ideas of topology and linear space theory in the calculus of variations, some unfortunate conflicts arise between the prevailing terminology in the respective fields. Let us state the position taken here with regard to two such conflicts. First, we apply the word closed both to surfaces (as well as to more general non-negative linear functionals $L$ ) and to subsets of topological spaces. Second, we use the term extreme point in the linear space sense and avoid use of extremum and its variants as they appear in the calculus of variations.

In addition to that introduced in $\S 1$, the following notation will be used throughout: $S^{m}$ denotes the set of skew symmetric $m \times m$ matrices of rank 0 or 2. Integrands $f(x, J)$ are assumed to be defined for $(x, J)$ in $R^{m} \times S^{m}$. For set sum and intersection, $\cup$ and $\cap$ are used. $|X|_{k}$ denotes the $k$-dimensional Lebesgue measure of a subset $X$ of $R^{m}$. The adjoint of a matrix $T$ is denoted by $T^{\prime}$. All sums are finite; the range of summation is omitted when the meaning is clear.

For an $m \times m$ matrix $J=\left[j^{r s}\right]$, let $\left.|J|=\mid \sum_{r, s}\left(j^{r s}\right)^{2}\right]^{1 / 2}$. Define $f_{a}$ in $E^{m}$ by the identity $f_{a}(x, J)=|J|$. For each non-negative linear functional $L$ on $E^{m}$, define the norm $a(L)=\left(L, f_{a}\right)$. If $L$ is a Dirichlet surface with Dirichlet representation $x(u, v)$ on the unit square $U$, and $J(u, v)$ is the Jacobian of $x(u, v)$, then 


$$
a(L)=\iint_{U}|J(u, v)| d u d v
$$

coincides with the Lebesgue area of the Fréchet surface with representation $x(u, v)$. If $x(u, v)$ satisfies a Lipschitz condition, the value for the area according to all reasonable definitions of area agrees with $a(L)$.

The modulus (or essential bound) $b(L)$ of $L$ is defined to be the smallest $b$ such that $L$ is situated in the sphere $|x| \leqq b$.

\section{PolyhedRa; EXACT INTEGRANDS}

3. Polyhedra; elementary boundary. Having introduced notions of surface and boundary in terms of linear functionals in $\$ 1$, we next define in these same terms an "orientation reversing" operation $(*)$. For each $f$, define $f^{*}$ by the identity $f^{*}(x, J)=f(x,-J)$. For each $L$, define $L^{*}$ by:

$$
\left(L^{*}, f\right)=\left(L, f^{*}\right) \text {. }
$$

If $L$ is a Dirichlet surface with Dirichlet representation $x(u, v)$, then $L^{*}$ has Dirichlet representation $x(1-u, v)$; i.e., $L^{*}$ is "oriented in the sense opposite to that of $L . "$

$L_{t}$ is termed a triangle if an oriented triangle $t$ in $R^{m}$ exists such that, for all $f,\left(L_{t}, f\right)$ is the value of the surface integral of $f$ over $t$. Let $t^{*}$ denote $t$ with opposite orientation. Then:

$$
L_{t}^{*}=L_{t^{*}} \text {. }
$$

$L$ is a polyhedron if $L$ is a sum of triangles. More generally, a linear combination with positive real coefficients of triangles $L_{t}$ is termed a polyhedron over the group $R$ of real numbers.

A mapping $x(u, v)$ from $U$ to $R^{m}$ is quasi-linear if $x(u, v)$ is linear in each triangle of a suitable finite subdivision of $U$. A quasi-linear mapping evidently defines a polyhedron $L$. It is easy to verify that, conversely, every polyhedron $L$ has a quasi-linear representation. Each polyhedron $L$ over $R$ may be written:

$$
L=\sum\left[a_{i} L_{t_{i}}+a_{i}^{*} L_{t_{i}}^{*}\right],
$$

where the $a_{i}, a_{i}^{*}$ are non-negative real numbers and the triangles $t_{i}$ meet at most along common edges and at common vertices. Such a decomposition is called a triangulation of $L$, and those $t_{i}\left(t_{i}^{*}\right)$ for which $a_{i}>0\left(a_{i}^{*}>0\right)$ are called faces of $L$. If $L$ is a polyhedron, the $a_{i}, a_{i}^{*}$ are integers, and conversely. If all $a_{i}=1$ and $a_{i}{ }^{*}=0$, then $L$ is said to be realized geometrically by the geometric polyhedron $P=\bigcup_{i} t_{i}$.

We next define an elementary notion of boundary as a weighted combination of bounding line segments, segments weighted equally but with opposite directions being allowed to cancel. This notion is made precise as follows: 
For each triangle $t$, let $\beta_{t}$ denote the linear functional on the space of forms $q=\sum q^{r}(x) d x^{r}$ with continuously differentiable coefficients $q^{r}$ whose value $\left(\beta_{t}, q\right)$ is, for each $q$, the value of the line integral of $q$ around the perimeter of $t$. Given a triangulation (3.1) of a polyhedron $L$ over $R$, define the elementary boundary (e-boundary) $\beta(L)$ of $L$ to be:

$$
\beta(L)=\sum\left[a_{i} \beta_{t_{i}}+a_{i}^{*} \beta_{t_{i}}^{*}\right] .
$$

By replacing any pair of triangulations by one finer than both, one finds that $\beta(L)$ is independent of the triangulation of $L$. Furthermore:

(3.2) The mapping $L \rightarrow \beta(L)$ preserves sums and positive scalar multiples. $\beta\left(L^{*}\right)=-\beta(L)$.

In other words, $\beta(L)$ behaves like the notion of boundary used in combinatorial topology. If $\beta=\beta(L)$ for some polyhedron $L$, then $\beta$ is called a closed polygonal path.

A polyhedron $L$ over $R$ is termed closed if $\beta(L)=0$ (this definition will be shown to agree with the definition $\lambda(L)=0$ of closed given in $\$ 1$ ). A polyhedron of the form $L_{t}+L_{t}^{*}$ is called a double triangle, since it corresponds geometrically to a pair of triangles back to back. A double triangle is evidently closed.

(3.3) Lemma. If a polyhedron $L$ over $R$ has e-boundary a closed polygonal path $\beta_{0}$, then $L$ is a linear combination with positive coefficients of polyhedra each of which has e-boundary a positive integral multiple of $\beta_{0}$.

Proof. Given a triangulation (3.1) of $L$, let $K$ denote the finite euclidean 2-complex (in the terminology of Lefschetz [9]) generated by the triangles $t_{i}$, i.e., which has as simplexes the $t_{i}$ plus their edges and vertices and orientation induced by that of the $t_{i}$. For $s=1,2$ and $G=R$ or $I$, the additive group of integers, let $C^{s}(K, G), Z^{s}(K, G)$ denote, respectively, the groups of $s$-chains and $s$-cycles over $K$ with coefficients in $G$. Let $\gamma(L)$ in $C^{2}(K, R)$ be defined by:

$$
\gamma(L)=\sum\left[a_{i} t_{i}+a_{i}^{*} t_{i}^{*}\right]=\sum\left[a_{i}-a_{i}^{*}\right] t_{i}
$$

where $a_{i}, a_{i}^{*}$ are as in (3.1).

Under the correspondence induced by identifying $\beta_{t}$ with the chain boundary $\partial t$ of a 2 -simplex $t$ of $K, \beta_{0}=\beta(L)$ corresponds to the chain boundary $\partial \gamma(L)$ of $\gamma(L)$. Since $\beta_{0}$ is a closed polygonal path, $\partial \gamma(L)$ is in $Z^{1}(K, I)$. Then since $\partial \gamma(L)$ bounds in $C^{2}(K, R)$, a positive integral multiple $\nu \partial \gamma(L)$ bounds some $\gamma_{1}$ in $C^{2}(K, I)$ (cf. Lefschetz [9, p. 106, (16.6)]). Since $\nu \gamma(L)-\gamma_{1}$ is in $Z^{2}(K, R)$, real coefficients $p_{j}$ and $z_{j}$ in $Z^{2}(K, I)$ exist [9, p. 105, (16.4)] such that:

$$
\nu \gamma(L)-\gamma_{1}=\sum p_{j} z_{j} .
$$

We may suppose all $p_{j}$ positive by changing signs of the $z_{j}$ as required. For 
each $\gamma=\sum \alpha_{i} t_{i}$, let:

$$
L_{\gamma}=\sum\left[\max \left(\alpha_{i}, 0\right) L_{t_{i}}+\max \left(-\alpha_{i}, 0\right) L_{t_{i}}^{*}\right] .
$$

Then from (3.4):

$$
L=L_{\gamma(L)}+\sum \dot{m}\left[\min \left(a_{i}, a_{i}^{*}\right)\right]\left[L_{t_{i}}+L_{t_{i}}^{*}\right] .
$$

In other words, $L_{\gamma(L)}$ is obtained by eliminating the double triangles from $L$. Since by (3.5)

$$
\gamma(L)=\nu^{-1} \gamma_{1}+\sum \nu^{-1} p_{j} z_{j},
$$

non-negative $k_{i}$ exist such that:

$$
L_{\gamma(L)}+\sum k_{i}\left(L_{t_{i}}+L_{t_{i}}^{*}\right)=\nu^{-1} L_{\gamma_{1}}+\sum \nu^{-1} p_{j} L_{z_{j}} .
$$

The double triangle terms on the left side of (3.7) appear because triangles with opposite orientation cancel when added in the combinatorial sense but add to form a double triangle when regarded as non-negative linear functionals.

We next obtain:

$$
L_{\gamma(L)}=\sum h_{j} L_{j},
$$

where the $h_{j}$ are positive and the $L_{j}$ are polyhedra bounded by non-negative integral multiples of $\beta_{0}$. To get (3.8), we eliminate the linear combination of double triangles on the left side of (3.7) by means of a suitable finite sequence of operations of the following type: Suppose that $L^{\prime}$ and $L^{\prime \prime}$ are polyhedra with $\beta\left(L^{\prime}\right)=\nu^{\prime} \beta_{0}, \beta\left(L^{\prime \prime}\right)=\nu^{\prime \prime} \beta_{0}$, where $\nu^{\prime}, \nu^{\prime \prime}$ are non-negative integers, and that $t_{i}, t_{i}^{*}$ are faces, respectively, of $L^{\prime}, L^{\prime \prime}$. Then if $0 \leqq h^{\prime} \leqq h^{\prime \prime}$,

$$
\begin{aligned}
& h^{\prime} L^{\prime}+h^{\prime \prime} L^{\prime \prime}-h^{\prime}\left(L_{t_{i}}+L_{t_{i}}^{*}\right) \\
& \quad=\left(h^{\prime \prime}-h^{\prime}\right) L^{\prime \prime}+h^{\prime}\left[L^{\prime}+L^{\prime \prime}-\left(L_{t_{i}}+L_{t_{i}}^{*}\right)\right] .
\end{aligned}
$$

The two polyhedra appearing on the right side of (3.9) are bounded by nonnegative integral multiples of $\beta_{0}$, and the coefficients $h^{\prime \prime}-h^{\prime}, h^{\prime}$ are nonnegative.

Having obtained (3.8) in this fashion, note next that, by (3.6), $L$ is likewise a positive linear combination of polyhedra bounded by non-negative multiples of $\beta_{0}$. By easy manipulations this sum may be rewritten so that no closed polyhedra appear, since closed polyhedra can be added to arbitrary polyhedra without changing the $e$-boundaries of the latter. This establishes (3.3).

4. Exact integrands. We use the term plane to denote an oriented, 2-dimensional, linear subset of $R^{m}$. Each plane $\pi$ has various linear representations $x(u, v)=x_{1} u+x_{2} v+x_{3}$. The Jacobian of $x(u, v)$ is the $m \times m$ matrix

$$
J_{x}=\left[x_{1}^{r} x_{2}^{s}-x_{1}^{s} x_{2}^{r}\right] \text {. }
$$


$J=\left|J_{x}\right|^{-1} J_{x}$ is independent of the linear representation $x(u, v)$ of $\pi$; we term $J$ the Jacobian of the plane $\pi$.

For $r, s=1,2, \cdots, m$, let

$$
J_{r s}=\left[\delta_{k r} \delta_{l s}-\delta_{k s} \delta_{l r}\right]
$$

denote the Jacobian of the $x^{r} x^{s}$ plane $\pi_{r s}$ ( $\delta_{i j}$ is Kronecker's delta). Under an affine transformation $x_{T}=T x+x_{0}$, the Jacobian $J$ changes according to $J_{T}=T J T^{\prime}$. Furthermore, it is well known that a necessary and sufficient condition that $J$ be skew-symmetric of rank 2 is that $T$ exist such that $J=T J_{12} T^{\prime}$, i.e., such that $|J|^{-1} J$ is the Jacobian of the plane $T \pi_{12}$. We have then:

(4.1) A necessary and sufficient condition that an $m \times m$ matrix $J$ be Jacobian of some plane $\pi$ is that $J$ be skew-symmetric of rank 2 and $|J|=1$. Two planes have the same Jacobian if and only if they differ by a translation in $R^{m}$.

Remark. For $m=3$, the vector $N=\left[j^{23}, j^{31}, j^{12}\right]$ is the positive unit normal to $\pi$, and $J$ may be identified with $N$.

Given a Dirichlet representation $x(u, v)=\left[x^{1}(u, v), \cdots, x^{m}(u, v)\right]$ with Jacobian $J(u, v)$, let $\left(u_{0}, v_{0}\right)$ be a point at which $J(u, v)$ exists and is not zero. Then the plane $\pi$ with linear representation $x_{u}\left(u_{0}, v_{0}\right) u+x_{v}\left(u_{0}, v_{0}\right) v+x\left(u_{0}, v_{0}\right)$ has Jacobian $\left|J\left(u_{0}, v_{0}\right)\right|^{-1} J\left(u_{0}, v_{0}\right)$. By (4.1), $J\left(u_{0}, v_{0}\right)$ is of rank 2. Thus:

(4.2) The range of the Jacobian $J(u, v)$ of a Dirichlet representation $x(u, v)$ lies in the set $S^{m}$ of skew-symmetric $m \times m$ matrices of rank 0 or 2 .

It also follows from (4.1) that every $J$ in $S^{m}$ is assumed by the Jacobian of some $x(u, v)$. This justifies our previous assumption that integrands $f$ are defined for $(x, J)$ in $R^{m} \times S^{m}$.

It is convenient to relax the requirement of continuity in $(x, J)$ originally made in the definition of exact integrand to suppose merely continuity in $x$ for each $J$. Since each $\phi$ exact according to the new definition will now be proved linear in $J$, the two definitions are equivalent.

(4.3) If $\phi$ is exact and $J_{1}, J_{2}, J_{1}+J_{2}$ are all in $S^{m}$, then $\phi\left(x, J_{1}\right)+\phi\left(x, J_{2}\right)$ $=\phi\left(x, J_{1}+J_{2}\right)$ for all $x$.

Proof. One may clearly suppose $J_{1}, J_{2} \neq 0$. Given $x$, let $\pi_{1}, \pi_{2}$ be planes through $x$ with Jacobians $\left|J_{1}\right|^{-1} J_{1},\left|J_{2}\right|^{-1} J_{2}$, respectively. Let $x(u, v)=x_{1} u$ $+x_{2} v+x$ be a linear representation of $\pi_{1}$. For $r=1,2, \cdots, m$, the $r$ th row vector $X_{r}$ of $J_{1}$ is a multiple of $x_{1}^{r} x_{2}-x_{2}^{r} x_{1}$. Thus, the $X_{r}$ lie in the linear 2 -space $\pi_{1}-x$ spanned by $x_{1}$ and $x_{2}$ (disregarding the orientation of $\pi_{1}$ for the present). Since $J_{1}$ is of rank 2 , the $X_{r}$ themselves span $\pi_{1}-x$. Letting $y_{1}, y_{2}$, $Y_{r}$ be similarly defined for $\pi_{2}$, the $Y_{r}$ span $\pi_{2}-x$.

Now $X_{r}+Y_{r}$ is the $r$ th row vector of $J_{1}+J_{2}$, for each $r=1,2, \cdots, m$. Since $J_{1}+J_{2}$ is in $S^{m}$, it is of rank at most 2; hence, there exist at least $m-2$ independent linear relations:

$$
\sum_{r=1}^{m} \alpha_{j r}\left(X_{r}+Y_{r}\right)=0 .
$$


Suppose $\sum \alpha_{j r} X_{r}=\sum \alpha_{j r} Y_{r}=0$ for all $j$. Then the $X_{r}, Y_{r}$ lie in a common 2 -space, which must by the remarks above coincide with both $\pi_{1}-x, \pi_{2}-x$. Hence, $\pi_{1}$ and $\pi_{2}$ coincide except for orientation in this case. If, on the other hand, for some $j$

$$
\sum \alpha_{j r} X_{r}=-\sum \alpha_{j r} Y_{r}=\bar{x} \neq 0,
$$

then $\bar{x}+x$ is in $\pi_{1} \cap \pi_{2}$ since the $X_{r}$ span $\pi_{1}-x$ and the $Y_{r}$ span $\pi_{2}-x$. In either case, $\pi_{1}$ and $\pi_{2}$ lie in at least one common 3 -dimensional linear subset $H$ of $R^{m}$.

We now define as follows a sequence $\left(L_{n}\right)$ of closed polyhedra with geometric realizations in $H$ and tending to $x: L_{n}$ is realized by a triangular prism whose lateral faces, respectively, have Jacobians $\left|J_{1}\right|^{-1} J_{1},\left|J_{2}\right|^{-1} J_{2}$, $-\left|J_{1}+J_{2}\right|^{-1}\left(J_{1}+J_{2}\right)$, and are of length $n^{-1}$ and width $n^{-2}\left|J_{1}\right|, n^{-2}\left|J_{2}\right|$, $n^{-2}\left|J_{1}+J_{2}\right|$. This construction is evidently valid for $m=3$ and follows for arbitrary dimension $m$ upon mapping $H$ onto $x^{1} x^{2} x^{3}$ space by a rigid motion, which preserves both norms and sums of matrices $J$. The sum of the areas of the bases of $L_{n}$ is then no more than $n^{-4}\left|J_{1}\right|\left|J_{2}\right|$. If $J_{1}=k J_{2}$, then all $L_{n}$ collapse into "double rectangles."

Since $\phi$ is exact and all $L_{n}$ are closed, $\left(L_{n}, \phi\right)=0$ for all $n$. Since $\phi$ is continuous in $x$ for each $J$, it follows that:

$$
0=\lim _{n} n^{3}\left(L_{n}, \phi\right)=\phi\left(x, J_{1}\right)+\phi\left(x, J_{2}\right)+\phi\left[x,-\left(J_{1}+J_{2}\right)\right] .
$$

Applying this first to the case $J_{2}=-J_{1}$, we get that $\phi(x,-J)=-\phi(x, J)$ for all $J$. Then in the general case:

$$
\begin{aligned}
0 & =\phi\left(x, J_{1}\right)+\phi\left(x, J_{2}\right)+\phi\left[x,-\left(J_{1}+J_{2}\right)\right] \\
& =\phi\left(x, J_{1}\right)+\phi\left(x, J_{2}\right)-\phi\left(x, J_{1}+J_{2}\right),
\end{aligned}
$$

which proves (4.3).

From (4.3) follows the linearity of $\phi$ in the variable $J$ :

(4.4) If $\phi$ is exact, then for all $x$ and $J=\left[j^{r s}\right]$ in $S^{m}$,

$$
\phi(x, J)=\sum_{r<s} \phi\left(x, J_{r s}\right) j^{r s}=\frac{1}{2} \sum_{r, s=1}^{m} \phi\left(x, J_{r s}\right) j^{r s} .
$$

(4.4) may be obtained easily by induction on $m$ from (4.3) using decompositions of the form $J=\sum_{s} J_{r s} j^{r s}+J_{1}$, where $J_{1}$ has all zeros in the $r$ th row and column.

If suitable differentiability conditions are satisfied, then exact integrands correspond to exterior derivatives of forms $q=\sum q^{r}(x) d x^{r}$ :

(4.5) If $\phi$ is exact and continuously differentiable, then a form $q=\sum q^{r}(x) d x^{r}$ with continuously differentiable coefficients $q^{r}(x)$ exists such that, for all $x$ and $J=\left[j^{r s}\right]$, 


$$
\phi(x, J)=\sum_{r<s}\left(q_{r}^{8}-q_{s}^{r}\right) j^{r s}
$$

Conversely, for each $q$ the corresponding $\phi$ defined by (4.6) is exact.

The first half of (4.5) follows from a theorem of De Rham, essentially to be found in Hodge [7, Chap. II]. The second half is a corollary of Stokes' theorem.

If one sets, in particular, $q^{s}(x)=x^{r}$ for $s=s_{1}$ and $q^{s}(x)=0$ for $s \neq s_{1}$, then (4.5) implies that $\phi_{r s}(x, J) \equiv j^{r s}$ is exact for each $r, s$. It follows by linearity that each linear combination

$$
\phi(J)=\sum_{r, s} \alpha_{r s} j^{r s}
$$

with constant coefficients $\alpha_{r s}$ is exact.

The following remark provides the basis for passing from the elementary notion of boundary introduced in $\$ 3$ for polyhedra to the notion of generalized boundary:

(4.7) Two polyhedra $L_{1}, L_{2}$ over $R$ have the same e-boundary if and only if $\left(L_{1}, \phi\right)=\left(L_{2}, \phi\right)$ for all exact $\phi$.

Proof. In view of (3.2) and (4.4), it suffices to show that $L_{1}+L_{2}^{*}$ is closed if and only if $\left(L_{1}+L_{2}^{*}, \phi\right)=0$ for all exact $\phi$. The necessity half of the latter assertion follows at once from the definition of exact and (3.3), while the sufficiency follows easily from Stokes' theorem.

5. A fundamental lemma. Lemma (5.1) is of fundamental importance in this paper; it is the key to the proofs given for the main theorems.

For each open subset $\Omega$ of $R^{m}$, let $B_{\Omega}$ denote the linear space consisting of all $\beta$ which bound polyhedra over $R$ situated in $\Omega$.

(5.1) Lemma. Let $\Omega \subset R^{m}$ be bounded, open, and convex. Then given $\beta_{0}$ in $B_{\Omega}$ and $f_{0}$ in $E^{m}$ such that the surface integral $\left(L, f_{0}\right)$ is non-negative for every closed polyhedron $L$ situated in $\Omega$, there exists a measurable function $\phi_{0}(x, J)$ and sequences $\left(\phi_{\nu}\right)$ of exact integrands and $\left(\rho_{\nu}\right)$ tending to 0 such that:

(1) The inf of $\left(L, f_{0}\right)$ in the set of all polyhedra $L$ over $R$ situated in $\Omega$ with $\beta(L)=\beta_{0}$ is $\left(L_{1}, \phi_{0}\right)$, where $L_{1}$ denotes any member of this set;

(2) $\phi_{0}(x, J)=\lim _{\nu} \phi_{\nu}(x, J)$ for almost all $x$;

(3) $\lim _{\nu}\left(L, \phi_{\nu}\right)=\left(L, \phi_{0}\right)$ for every polyhedron $L$ over $R$; and, for each $\nu=1,2, \cdots$,

(4) $\phi_{\nu}(x, J) \leqq f_{0}(x, J)+\rho_{\nu}|J|$ for $(x, J)$ in $\Omega \times S^{m}\left(^{4}\right)$.

Proof. For $\beta$ in $B_{\Omega}$, let

$$
\mu(\beta)=\inf \left(L, f_{0}\right), \quad\|\beta\|_{\Omega}=\inf a(L),
$$

where the inf is in each case taken in the set of all polyhedra $L$ over $R$ situated in $\Omega$ with $\beta(L)=\beta$. Let $\|\beta\|$ denote the corresponding inf for $\Omega=R^{m}$. It is easy to verify that $\|\beta\|_{\Omega}$ and $\|\beta\|$ have the usual norm properties. Since the

(4) For a corresponding result for curves, see [5]. 
set $\Omega$ in (5.1) is convex, $\|\beta\|=\|\beta\|_{\Omega}$ for $\beta$ in $B_{\Omega}$.

By (3.3), every closed polyhedron $L$ over $R$ is a positive linear combination of closed polyhedra. It then follows from the hypotheses of (5.1) that $\left(L, f_{0}\right) \geqq 0$ for every closed polyhedron $L$ over $R$ situated in $\Omega$. Thus $\mu(0) \geqq 0$; since there are closed polyhedra of arbitrarily small norm situated in $\Omega$, $\mu(0)=0$. For all $\beta, \beta^{\prime}$ in $B_{\Omega}$ :

$$
\mu\left(\beta+\beta^{\prime}\right) \leqq \mu(\beta)+\mu\left(\beta^{\prime}\right) \text { and } \mu(k \beta)=k \mu(\beta) \quad \text { for } k \geqq 0 .
$$

Since $0=\mu(0) \leqq \mu(\beta)+\mu(-\beta), \mu(\beta)$ is finite for all $\beta$ in $B_{\Omega}$. Then by the well known Hahn-Banach theorem [2, p. 29], a linear functional $l$ on $B_{\Omega}$ exists such that $(l, \beta) \leqq \mu(\beta)$ for all $\beta$ in $B_{\Omega}$ and $\left(l, \beta_{0}\right)=\mu\left(\beta_{0}\right)$. Let

$$
M=\sup _{x \in \Omega,|J|=1}\left|f_{0}(x, J)\right| .
$$

For each $\beta$ in $B_{\Omega}$ and $L$ situated in $\Omega$ with $\beta(L)=\beta$,

$$
(l, \beta) \leqq \mu(\beta) \leqq\left(L, f_{0}\right) \leqq M a(L),
$$

and

$$
-(l, \beta)=(l,-\beta) \leqq \mu(-\beta) \leqq\left(L^{*}, f_{0}\right) \leqq M a\left(L^{*}\right)=M a(L) .
$$

It follows that for $\beta$ in $B_{\Omega},|(l, \beta)| \leqq M\|\beta\|_{\Omega}$, or since $\|\beta\|=\|\beta\|_{\Omega}$,

$$
|(l, \beta)| \leqq M\|\beta\| \text {. }
$$

By a corollary of the Hahn-Banach theorem [2, p. 55], $l$ has an extension to the space of all $\beta$ such that (5.2) continues to hold.

Let $J_{0}$ in $S^{m}$ with $\left|J_{0}\right|=1$ be given; let $\pi_{0}$ denote the plane through $x=0$ with Jacobian $J_{0}$, and $T$ the matrix of an orthogonal transformation carrying the $x^{1} x^{2}$ plane $\pi_{12}$ into $\pi_{0}$. Let $V_{1}, \cdots, V_{m}$ denote the column vectors of $T$. For each $p=\left[p^{3}, \cdots, p^{m}\right]$, let

$$
x_{p}(u, v)=V_{1} u+V_{2} v+\sum_{s=3}^{m} V_{s} p^{s},
$$

and $\pi_{p}$ the plane which has linear representation $x_{p}(u, v)$. The planes $\pi_{p}$ cover $R^{m}$ and have Jacobian $J_{0}$ (cf. $\$ 4$ ).

For each $u v$ interval $\Delta$ and each $p$, let $L_{\Delta, p}$ denote the polyhedron realized geometrically by the rectangle $\Delta_{p}=x_{p}(\Delta)$, in $\pi_{p}$. Let $\beta_{\Delta, p}$ denote the $e$-boundary of $L_{\Delta, p}$ and

$$
F_{p}(\Delta)=\left(l, \beta_{\Delta, p}\right) .
$$

By (5.2) and the orthogonality of $T$,

$$
\left|F_{p}(\Delta)\right| \leqq M\left|\Delta_{p}\right|_{2}=M|\Delta|_{2}
$$

for all $\Delta$. Moreover, $F_{p}$ is an additive function of intervals $\Delta$, since $l$ is an 
additive function of boundaries $\beta$. Consequently, for each $p$ the derivative $D_{p}(u, v)$ of $F_{p}$ exists for almost all $(u, v)$, and for all $\Delta$ (cf. Saks [12, Chap. IV]),

$$
F_{p}(\Delta)=\iint_{\Delta} D_{p}(u, v) d u d v .
$$

The choice of $J_{0}$ of norm 1 was arbitrary; hence, we write $D_{p}(u, v)$ as a function $D_{p}[u, v ; J]$ of $J$ as well as $(u, v)$. Define $\phi_{0}(x, J)$ for $x=x_{p}(u, v)$ in $\pi_{p}$ and $|J|=1$ by:

$$
\phi_{0}\left[x_{p}(u, v), J\right]=\left\{\begin{array}{lr}
D_{p}[u, v ; J], & \text { if } D_{p}[u, v ; J] \text { exists; } \\
f_{0}\left[x_{p}(u, v), J\right], & \text { otherwise. }
\end{array}\right.
$$

The latter definition applies for each $p$ only in a subset of $\pi_{p}$ of measure 0 ; hence, by Fubini's theorem, it applies only for a subset of $R^{m}$ of $m$-dimensional measure 0 . The definition of $\phi_{0}$ is extended by homogeneity to $J$ of arbitrary norm.

We next observe that, for all polyhedra $L$ over $R$,

$$
\left(L, \phi_{0}\right)=(l, \beta(L)) .
$$

For, (5.5) follows for triangles easily from the definition of $\phi_{0},(5.2)$, and (5.4). It then follows by linearity for all polyhedra over $R$. Assertion (1) of (5.1) is now an immediate consequence of (5.5) and the fact that $\left(l, \beta_{0}\right)=\mu\left(\beta_{0}\right)$.

To show that $\phi_{0}$ is measurable, let $\left(N_{v}\right)$ denote a binary sequence [12, p. 191] of nets $N_{\nu}$ of half-open $u v$ intervals $\Delta$, and $J_{0}$ a fixed element of $S^{m}$ with $\left|J_{0}\right|=1$. For each $\nu=1,2, \cdots$ and $\Delta$ in $N_{\nu}$, let

$$
h_{\nu}(x)=\frac{\left(l, \beta_{\Delta, p}\right)}{|\Delta|_{2}}
$$

for $x$ in $\Delta_{p}=x_{p}(\Delta)$. Now $h_{\nu}(x)$ is continuous in the interior of the block $D_{\Delta}=\cup_{p} \Delta_{p}$. To see this, consider $x \in \Delta_{p}, x^{\prime} \in \Delta_{p^{\prime}}$ in $D_{\Delta}$, and let $L$ denote the polyhedron realized by joining corresponding corners of the rectangles $\Delta_{p}, \Delta_{p^{\prime}}$. Since $T$ is orthogonal,

$$
a(L)=\sigma\left|p-p^{\prime}\right| \leqq \sigma\left|x-x^{\prime}\right|
$$

where $\sigma$ denotes the length of the perimeter of $\Delta$.

$$
\beta(L)= \pm\left(\beta_{\Delta, p}-\beta_{\Delta, p^{\prime}}\right) .
$$

Hence,

$$
\begin{aligned}
|\Delta|_{2}\left|h_{\nu}(x)-h_{\nu}\left(x^{\prime}\right)\right| & =\left|\left(l, \beta_{\Delta, p}\right)-\left(l, \beta_{\Delta, p^{\prime}}\right)\right|=\left|\left(l, \beta_{\Delta, p}-\beta_{\Delta, p^{\prime}}\right)\right| \\
& =|(l, \beta(L))| \leqq M a(L) \leqq M \sigma\left|x-x^{\prime}\right|
\end{aligned}
$$

and $h_{\nu}(x)$ is Lipschitzian and therefore continuous in $D_{\Delta}$. 
Since $h_{\nu}(x)$ is thus piecewise continuous for $\nu=1,2, \cdots$ and

$$
\lim _{\nu} h_{\nu}(x)=\phi_{0}\left(x, J_{0}\right)
$$

for almost all $x, \phi_{0}\left(x, J_{0}\right)$ is measurable. Since $J_{0}$ is arbitrary and $\phi_{0}$ will now be proved linear in $J, \phi_{0}$ is measurable in $(x, J)$.

We may now define for $\nu=1,2, \cdots$ and all $J$ in $S^{m}$ the mean value

$$
\phi_{\nu}(x, J)=\frac{1}{\left|D_{\nu}\right|_{m}} \int_{D_{\nu}} \phi_{0}\left(x+x^{\prime}, J\right) d x^{\prime}
$$

over a cube $D_{\nu}$ in $R^{m}$ with center 0 and diameter $\nu^{-1}$. By (5.2) and the definition of $\phi_{0},\left|\phi_{0}(x, J)\right| \leqq M|J|$ for all $(x, J)$; hence, for all $\nu=1,2, \cdots$ and all $(x, J)$ :

$$
\left|\phi_{\nu}(x, J)\right| \leqq M|J| \text {. }
$$

Let $L$ be a closed polyhedron with quasi-linear representation $x(u, v)$. Then for every $x^{\prime}, x(u, v)+x^{\prime}$ is a quasi-linear representation with the same Jacobian as $x(u, v)$ of a closed polyhedron $L^{\prime}$ (the translate of $L$ by $\left.x^{\prime}\right)$. Using this remark, Fubini's theorem, and the fact that by $(5.5)\left(L^{\prime}, \phi_{0}\right)=0$ for all such $L^{\prime}$, one finds that $\left(L, \phi_{\nu}\right)=0$ for all $\nu$. Since each $\phi_{\nu}$ is a continuous function of $x$ and $L$ is arbitrary, each $\phi_{\nu}$ is exact (cf. the definition of exact given in $\S 4) . \phi_{\nu}(x, J)$ converges to $\phi_{0}(x, J)$ for almost all $x$ given $J$, and hence simultaneously for $J$ in a countable dense subset of $S^{m}$ except for a fixed null set $N$ of $x$. Then, by (5.2), $\phi_{\nu}(x, J)$ converges to $\phi_{0}(x, J)$ for all $J$ and $x$ not in $N$. Thus, (2) of (5.1) holds.

For $\nu=1,2, \cdots$, define linear functionals $l_{\nu}$ by the equation

$$
\left(l_{\nu}, \beta(L)\right)=\left(L, \phi_{\nu}\right) .
$$

From (5.7) it follows that, for all $\beta$,

$$
\left|\left(l_{\nu}, \beta\right)\right| \leqq M\|\beta\| \text {. }
$$

Let $J_{0}$ of norm 1 be given, and $x_{p}, \pi_{p}$ as before. The sequence $\phi_{\nu}\left(x, J_{0}\right)$ converges to $\phi_{0}\left(x, J_{0}\right)$ almost everywhere in $R^{m}$ and hence, except for a set $H$ of $p$ such that $|H|_{m-2}=0$, almost everywhere in $\pi_{p}$. It then follows from the uniform boundedness (5.7) of the $\phi_{\nu}$ that for every triangle $t$ in $\pi_{p}$ for $p$ not in $H$,

$$
\begin{aligned}
\left(l_{v}, \beta_{t}\right) & =\iint_{x_{\bar{p}^{1}}(t)} \phi_{\nu}\left[x_{p}(u, v), J_{0}\right] d u d v \\
& \rightarrow \iint_{x_{\bar{p}^{1}}(t)} \phi_{0}\left[x_{p}(u, v), J_{0}\right] d u d v=\left(l, \beta_{t}\right)
\end{aligned}
$$


as $\nu \rightarrow \infty$. It follows from (5.8) that (5.9) holds for arbitrary $p$, since an arbitrary $t$ with direction $J_{0}$ can be approximated in the \|\| sense by a sequence of triangles in planes $\pi_{p}$ for $p$ not in $H$. Since $J_{0}$ is arbitrary, assertion (3) of (5.1) follows by linearity and (5.5).

By the definition of $\phi_{0}, l$, and $\mu$, one has for $x=x_{p}(u, v)$ in $\Omega$ :

$$
\phi_{0}\left[x_{p}(u, v), J_{0}\right]=\lim _{\Delta \rightarrow(u, v)} \frac{\left(l, \beta_{\Delta, p}\right)}{|\Delta|_{2}} \leqq \lim _{\Delta \rightarrow(u, v)} \frac{\left(L_{\Delta, p}, f_{0}\right)}{|\Delta|_{2}}=f_{0}\left[x_{p}(u, v), J_{0}\right] \text {, }
$$

whenever the first limit appearing in (5.10) exists, and is equal to $f_{0}$ otherwise. Thus, since $J_{0}$ is arbitrary, $\phi_{0}(x, J) \leqq f_{0}(x, J)$ for all $(x, J)$ in $\Omega \times S^{m}$. For $\nu=1,2, \cdots$, let

$$
\rho_{\nu}=\sup _{x^{\prime}-x \in D_{\nu}, x \in \Omega,|J|=1}\left|f_{0}\left(x^{\prime}, J\right)-f_{0}(x, J)\right| .
$$

Since $\Omega$ is bounded and $f_{0}$ is uniformly continuous on bounded sets, $\rho_{\nu} \rightarrow 0$ as $\nu \rightarrow \infty$. For all $\nu$ and $(x, J)$ in $\Omega \times S^{m}$,

$$
\phi_{\nu}(x, J) \leqq \sup _{x^{\prime} \in x+D_{\nu}} \phi_{0}\left(x^{\prime}, J\right) \leqq \sup _{x^{\prime} \in x+D_{\nu}} f_{0}\left(x^{\prime}, J\right) \leqq f_{0}(x, J)+\rho_{\nu}|J|,
$$

which proves (4) of (5.1).

REMARK. It probably is not true that in (5.1) $\phi_{0}$ may be supposed continuous. Damköhler [4] has given a counterexample in a corresponding problem for curves.

\section{$G$-BOUNDARIES}

6. G-boundaries and Dirichlet surfaces. We are now ready to consider the general notions of boundary and track (especially boundary) introduced in $\S 1$. From the definitions and the linearity in the variable $J$ of exact integrands, established in (4.4), it follows at once that:

(6.1) The generalized boundary and track are preserved under addition and positive scalar multiplication, and change sign under the operation (*). If $L_{1}$ and $L_{2}$ have the same generalized track, they have the same generalized boundary. The $w^{*}$ limit of a sequence with fixed generalized boundary or track has again that generalized boundary or track.

In view of (4.7) the notion of generalized boundary (abbreviated, $g$-boundary) is an extension of the notion of $e$-boundary defined in $\$ 3$ for polyhedra; i.e., the g-boundary is a generalization of line integration around a boundary curve, paths traversed in opposite senses being allowed to cancel.

We are interested here primarily in admissible $g$-boundaries. An admissible $g$-boundary $\lambda$ is termed rectifiable, or a closed polygonal path, if it bounds some Dirichlet surface with a Dirichlet representation $x(u, v)$ which reduces on the perimeter of $U$ to a representation of a Fréchet curve with the named property.

If $x(u, v)$ is a Dirichlet representation of a Dirichlet surface $L$, a new 
representation of $L$ may be found which is constant on one side of $U$. One gets such a representation, for instance, by choosing a Lipschitzian mapping $u=u(\xi, \eta), v=v(\xi, \eta)$ of $U$ into itself which is constant on $\xi=1$ and biunique otherwise, and setting $x^{\prime}(\xi, \eta)=x[u(\xi, \eta), v(\xi, \eta)]$. According to [17, p. 325, (11.2)], $x^{\prime}(\xi, \eta)$ is a Dirichlet representation and the partial derivatives of $x^{\prime}$ are given almost everywhere by the usual formula. It follows from a well known change of variables theorem and the homogeneity in $J$ of integrands $f$ that, for all $f$ in $E^{m}$,

$$
(L, f)=\iint_{U} f[x(u, v), J(u, v)] d u d v=\iint_{U} f\left[x^{\prime}(\xi, \eta), J^{\prime}(\xi, \eta)\right] d \xi d \eta ;
$$

i.e., $x^{\prime}$ is also a Dirichlet representation of $L$.

Using this remark, it is easy to "join two Dirichlet surfaces with a thread" and get:

(6.2) If $L_{1}$ and $L_{2}$ are Dirichlet surfaces, so is $L_{1}+L_{2}$.

Dirichlet surfaces bounded in the sense of classical analysis by the same rectifiable curve $C$ have the same $g$-boundary:

(6.3) If $L_{1}, L_{2}$ have, respectively, Dirichlet representations $x_{1}(u, v), x_{2}(u, v)$ which reduce on the perimeter of $U$ to representations of the same rectifiable closed Fréchet curve $C$, then $\lambda\left(L_{1}\right)=\lambda\left(L_{2}\right)\left({ }^{5}\right)$.

(6.3) is a trivial consequence of (4.5), Stokes' theorem, and the fact that every exact integrand $\phi$ is the limit uniformly on bounded sets of $(x, J)$ of a sequence of continuously differentiable exact integrands (obtained, for instance, by approximating $\phi$ by mean value integrals with respect to the variable $x$ ).

If the condition of rectifiability is dropped, (6.3) does not always hold. For instance, let $C$ be a simple closed curve in the unit square in the $x^{1} x^{2}$ plane of $R^{3}$ such that the set [C] occupied by $C$ has positive 2-dimensional measure $\mu$. Let $L_{0}$ denote the Dirichlet surface realized by the surface $\Sigma$ of the unit cube in $R^{3}$ (with orientation that of $C$ ); $L_{1}$, the smaller component of $\Sigma-[C]$; and $L_{2}$, the larger component. $L_{1}$ and $L_{2}^{*}$ are Dirichlet surfaces with $C$ as boundary curve in the classical sense. Consider the exact integrand $\phi_{12}(x, J) \equiv j^{12}$. Now

$$
\left(L_{0}-\left(L_{1}+L_{2}\right), \phi_{12}\right)= \pm \iint_{[C]} d x^{1} d x^{2},
$$

the sign being positive if the orientation of $C$ is positive. Since $L_{0}$ is closed, $\left(L_{0}, \phi_{12}\right)=0$; hence,

$$
\left(L_{1}, \phi_{12}\right)=\left(L_{2}^{*}, \phi_{12}\right) \pm \mu .
$$

Thus $\lambda\left(L_{1}\right) \neq \lambda\left(L_{2}^{*}\right)$.

(5) (Added in proof.) Using recent results of Young, as yet unpublished, (6.3) can also be proved for nonrectifiable curves $C$ whose set of points is of 2-dimensional Hausdorff measure 0 . 
(6.4) Given $\epsilon>0$ and a Dirichlet surface $L$ situated in an open set $\Omega$, there exist a closed polygonal path $\lambda_{\epsilon}$ and a Dirichlet surface $L_{\epsilon}$ situated in $\Omega$ with $a\left(L_{\epsilon}\right)<\epsilon$ and $\lambda\left(L_{\epsilon}\right)=\lambda(L)-\lambda_{\epsilon}$.

The proof of (6.4) is elementary if $\lambda(L)$ is rectifiable. To establish the general case, it then suffices to prove (6.4) with $\lambda_{\epsilon}$ rectifiable. Let $x(u, v)$ be a Dirichlet representation of $L$. Since $x(u, v)$ is absolutely continuous in Tonelli's sense, $x\left(u_{0}, v\right)$ and $x\left(u, v_{0}\right)$ define rectifiable curves for sets $u_{0}$ and $v_{0}$ dense, respectively, in $(0,1)$. Choose a rectangle $U^{\prime}:\left[u_{1} \leqq u \leqq u_{2} ; v_{1} \leqq v \leqq v_{2}\right]$, where $u_{1}, u_{2}$ and $v_{1}, v_{2}$ lie, respectively, in these sets, such that $\iint_{U-U^{\prime}}|J(u, v)|$ $<\epsilon$. Let $L_{\epsilon}$ be the Dirichlet surface defined by the representation $x(u, v)$ on $U-U^{\prime} ;$ let $\lambda_{\epsilon}=\lambda(L)-\lambda\left(L_{\epsilon}\right)$.

7. Non-negative linear $L$ with admissible $g$-boundary. For each $A \subset R^{m}$, let $E^{m, A}$ denote the linear space of restrictions of integrands $f(x, J)$ in $E^{m}$ to values $(x, J)$ in $A \times S^{m}$. If $A$ is bounded, $E^{m, A}$ is a Banach space under the norm

$$
\|f\|_{A}=\sup _{x \in A,|J|=1}|f(x, J)|
$$

The set of non-negative linear $L$ situated in the closure of $A$ may be identified in the obvious fashion with the set of non-negative elements of the conjugate of $E^{m, A}$, and $a(L)$ coincides with the usual conjugate space norm. We further define in this set the McShane distance [10, p. 534]:

$$
\left\|L_{1}, L_{2}\right\|_{A}=\sup _{f \in F_{A}}\left|\left(L_{1}, f\right)-\left(L_{2}, f\right)\right|,
$$

where $F_{A}$ denotes the set of $f$ in $E^{m, A}$ such that $\|f\|_{A}=1$ and $\left|f(x, J)-f\left(x^{\prime}, J^{\prime}\right)\right|$ $\leqq\left(\left|x-x^{\prime}\right|^{2}+\left|\left(J-J^{\prime}\right)\right|^{2}\right)^{1 / 2}$. In the set of non-negative linear $L$ situated in $A$, the McShane distance defines a metric topology equivalent to the $w^{*}$ topology. This follows from the known equivalence of convergence in the McShane distance with $w^{*}$ convergence for sequences of elements of this set, and the fact that from a $w^{*}$ convergent directed sequence $\left(L_{\alpha}\right)$ of non-negative linear functionals situated in a fixed bounded set one can extract a sequence in the ordinary sense converging $w^{*}$ to the same limit. The latter assertion follows from the separability of $E^{m, A}$, and the fact that $a(L)=\left(L, f_{a}\right)$ for $L$ non-negative which implies that a bounded directed subsequence of $\left(L_{\alpha}\right)$ can be found.

We are now prepared to apply the results of the preceding sections to characterize the set of non-negative linear $L$ which have an admissible g-boundary.

(7.1) Lemma. Let $\Omega \subset R^{m}$ be bounded, open, and convex. Let $\lambda_{0}$ bound some polyhedron over $R$ situated in $\Omega$. Then every non-negative linear $L_{0}$ situated in $\Omega$ with $\lambda\left(L_{0}\right)=\lambda_{0}$ is the $w^{*}$ limit of a sequence $\left(L_{n}\right)$ of polyhedra over $R$ situated in $\Omega$ such that $\lambda\left(L_{n}\right)=\lambda_{0}$ for $n=1,2, \cdots$. 
Proof. By hypothesis, $\lambda_{0}=\lambda(L)$ for some polyhedron $L$ over $R$ situated in $\Omega$. Then $\beta_{0}=\beta(L)$ is in the set $B_{\Omega}$ defined in $\S 5$. The set $\Gamma$ of all polyhedra over $R$ situated in $\Omega$ and bounded by non-negative multiples of $\beta_{0}$ is a convex cone in the conjugate of the Banach space $E^{m, \Omega}$. We show first that $L_{0}$ is in the $w^{*}$ closure of $\Gamma$.

Suppose this is not the case. Then by the well known separation theorem for $w^{*}$ closed, convex subsets of the conjugate of a Banach space, $L_{0}$ can be separated from $\Gamma$ by a hyperplane. This means that $f_{0}$ in $E^{m, \Omega}$ and $\alpha$ exist such that $\left(L_{0}, f_{0}\right)<\alpha$ and $\left(L, f_{0}\right) \geqq \alpha$ for all $L$ in $\Gamma$. Since $\Gamma$ is a cone, $\alpha=0$. Since $f_{0}$ is the restriction of an integrand in $E^{m}$, we regard $f_{0}$ itself as being in $E^{m}$.

Every closed polyhedron situated in $\Omega$ is in $\Gamma$; hence, the hypotheses of Lemma (5.1) are satisfied. Let $\phi_{0},\left(\phi_{\nu}\right),\left(\rho_{v}\right)$, and $L_{1}$ be as in (5.1). By (1) of (5.1) and hypothesis, $\left(L_{1}, \phi_{0}\right) \geqq 0$. Then using the fact that $\lambda\left(L_{1}\right)=\lambda\left(L_{0}\right)$ and (3) of (5.1):

$$
\begin{aligned}
\lim _{\nu}\left(L_{0}, f_{0}-\phi_{\nu}+\rho_{\nu} f_{a}\right) & =\left(L_{0}, f_{0}\right)-\lim _{\nu}\left(L_{0}, \phi_{\nu}\right) \\
& =\left(L_{0}, f_{0}\right)-\lim _{\nu}\left(L_{1}, \phi_{\nu}\right)=\left(L_{0}, f_{0}\right)-\left(L_{1}, \phi_{0}\right)<0 .
\end{aligned}
$$

But $f_{0}-\phi_{\nu}+\rho_{\nu} f_{a} \geqq 0$ for all $\nu$; hence, $\left(L_{0}, f_{0}-\phi_{\nu}+\rho_{\nu} f_{a}\right) \geqq 0$ for all $\nu$ since $L_{0}$ is non-negative, a contradiction.

Therefore, $L_{0}$ is a $w^{*}$ limit point of $\Gamma$. Since $\Omega$ is bounded, the $w^{*}$ topology is metrizable by the McShane distance in the set of non-negative linear $L$ situated in $\Omega$. Hence, a sequence $\left(L_{n}\right)$ in $\Gamma$ converges $w^{*}$ to $L_{0}$. For all $n$ $=1,2, \cdots, \lambda\left(L_{n}\right)=k_{n} \lambda_{0}$, where $k_{n} \geqq 0$. For all exact $\phi$,

$$
\left(\lambda_{0}, \phi\right)=\left(\lambda\left(L_{0}\right), \phi\right)=\lim _{n}\left(\lambda\left(L_{n}\right), \phi\right)=\lim _{n} k_{n}\left(\lambda_{0}, \phi\right) .
$$

Then either $\lambda_{0}=0$ or else $k_{n} \rightarrow 1$, in which case $k_{n}^{-1} L_{n}$ converges $w^{*}$ to $L_{0}$ and $\lambda\left(k_{n}^{-1} L_{n}\right)=\lambda_{0}$ for all $n$. In either case, the proof of (7.1) is complete.

We now prove the first half of Theorem (1.1):

(7.2) Let $\lambda_{0}$ be admissible. Then a necessary and sufficient condition that $L_{0}$ have g-boundary $\lambda\left(L_{0}\right)=\lambda_{0}$ is that $L_{0}$ be the w* limit of a sequence of linear combinations $\sum k_{j} L_{j}$ with positive coefficients $k_{j}$ of Dirichlet surfaces $L_{j}$ with $\lambda\left(L_{j}\right)$ $=\nu_{j} \lambda_{0}$, where each $\nu_{j}$ is a positive integer and $\sum k_{j} \nu_{j}=1$.

Proof. The sufficiency is an immediate consequence of the definitions of $g$-boundary and $w^{*}$ convergence. In proving the necessity, suppose first that $\lambda_{0}$ is a closed polygonal path. By Lemma (7.1), $L_{0}=w^{*} \lim _{n} L_{n}$, where each $L_{n}$ is a polyhedron over $R$ with $\lambda\left(L_{n}\right)=\lambda_{0}$. Applying (3.3), each $L_{n}$ is of the form $\sum k_{j} L_{j}$, where the $k_{j}$ are positive real coefficients and the $L_{j}$ are polyhedra with each $\lambda\left(L_{j}\right)$ a positive integral multiple $\nu_{j} \lambda_{0}$ of $\lambda_{0}$. Thus, to prove (7.2) for closed polygonal paths $\lambda_{0}$ it suffices to show that one may suppose $\sum k_{j} \nu_{j}=1$. 
If $\lambda_{0} \neq 0$, the argument used in the last paragraph of the proof of (7.1) shows that $\eta=\sum k_{j} \nu_{j} \rightarrow 1$; replace each $k_{j}$ by $\eta^{-1} k_{j}$.

Suppose $\lambda_{0}=0$. Then since the $L_{j}$ are closed polyhedra, so are $N L_{j}$ for all positive integers $N$ and all $j$. Choose all $\nu_{j}=1$, and $N>\sum k_{j}$. Replacing $\sum k_{j} L_{j}$ by $\sum\left(N^{-1} k_{j}\right)\left(N L_{j}\right)+\left[1-\sum\left(N^{-1} k_{j}\right)\right] L^{\prime}$, where $L^{\prime}$ is a closed polyhedron of small area, establishes that for $\lambda_{0}=0$ one may suppose $\sum k_{j} \nu_{j}$ $=\sum k_{j}=1$.

Having proved (7.2) for closed polygonal paths, now let $\lambda_{0}$ be an arbitrary admissible $g$-boundary. Let $L$ be a Dirichlet surface with $\lambda(L)=\lambda_{0}$, and $\Omega$ a bounded open convex set in which both $L$ and $L_{0}$ are situated. Given $\epsilon>0$ let $\lambda_{\epsilon}$ and $L_{\epsilon}$ be as in (6.4). Then

$$
\lambda\left(L_{0}+L_{\epsilon}^{*}\right)=\lambda_{\epsilon} .
$$

By what has already been proved, $L_{0}+L_{\epsilon}^{*}$ is the $w^{*}$ limit of sums $\sum k_{j} L_{j}$, where $\lambda\left(L_{j}\right)=\nu_{j} \lambda_{\epsilon}$ for all $j$ and $\sum k_{j} \nu_{j}=1$ as above. The $L_{j}$ may be assumed to be situated in $\Omega$. Then

$$
L_{0}+L_{\epsilon}^{*}+L_{\epsilon}=w^{*} \lim \sum k_{j}\left(L_{j}+\nu_{j} L_{\epsilon}\right),
$$

and each $L_{j}+\nu_{j} L_{\epsilon}$ is a Dirichlet surface with

$$
\lambda\left(L_{j}+\nu_{j} L_{\epsilon}\right)=\nu_{j} \lambda_{0} .
$$

Hence (7.2) holds for $L_{0}+L_{\epsilon}^{*}+L_{\epsilon}$. Since the set of non-negative linear $L$ situated in $\Omega$ is metrizable in a way equivalent to $w^{*}$ convergence and $a\left(L_{\epsilon}\right)$ $+a\left(L_{\epsilon}^{*}\right)<2 \epsilon,(7.2)$ follows for $L_{0}$ upon letting $\epsilon \rightarrow 0$.

8. Completion of the proof of Theorem (1.1). The first half of Theorem (1.1) was established in (7.2). It remains to show that for $m=3$, one can suppose in (7.2) that all $\nu_{j}=1$, i.e., that the set of non-negative linear $L$ with admissible $g$-boundary consists of all $w^{*}$ limits of convex combinations of Dirichlet surfaces with that $g$-boundary. To do this, we prove first:

(8.1) Suppose $m=3$. Let $L_{0}$ be a polyhedron with e-boundary $\beta\left(L_{0}\right)=\nu \beta_{0}$, where $\beta_{0} \neq 0$ is a closed polygonal path and $\nu$ is a positive integer. Then poly. hedra $L_{1}, \cdots, L_{\nu}$ exist with $\beta\left(L_{1}\right)=\cdots=\beta\left(L_{\nu}\right)=\beta_{0}$ such that $L_{0}=\sum L_{j}$.

Proof. It suffices to suppose that $L_{0}$ has no double triangles, i.e., that for no triangle $t$ do both $t$ and $t^{*}$ appear as faces of $L_{0}$, since every polyhedron reduces to such a polyhedron upon subtracting a sum of double triangles (which is closed). Then $L_{0}$ has a triangulation

$$
L_{0}=\sum n_{i} L_{t_{i}}
$$

with all $n_{i}$ positive integers. As in the proof of (3.3), let $K$ denote the euclidean 2-complex in $R^{3}$ generated by the $t_{i}$; let

$$
\gamma\left(L_{0}\right)=\sum n_{i} t_{i} .
$$

The closed polygonal path $\beta_{0}$ corresponds to $\zeta=\nu^{-1} \partial \gamma\left(L_{0}\right)$ in $Z^{1}(K, I)$. Since 
$K$ is in $R^{3}, Z^{1}(K, I)$ contains no torsion cycles (cf. Alexandroff-Hopf [1, p. 390]); hence, $\zeta=\partial \gamma$ for some $\gamma$ in $C^{2}(K, I)$ since $\nu \zeta$ bounds in $C^{2}(K, I)$. Then $\gamma\left(L_{0}\right)=\nu \gamma+z$, where $z$ is in $Z^{2}(K, I)$. Since $L_{0}$ has no double triangles, we have as in (3.7),

$$
L_{0}+\sum h_{i}\left(L_{t_{i}}+L_{t_{i}}^{*}\right)=\nu L_{\gamma}+L_{z}=(\nu-1) L_{\gamma}+\left(L_{\gamma}+L_{z}\right),
$$

for suitable non-negative integers $h_{i}$. To complete the proof of (8.1), the sum of double triangles in (8.2) will be eliminated in $\sum h_{i}$ successive steps. The means for doing this will be the result that if $t$ is a face of $L_{1}, t^{*}$ a face of $L_{2}$, and $\beta\left(L_{1}\right)=\beta\left(L_{2}\right)=\beta_{0}$, then polyhedra $L_{1}^{\prime}, L_{2}^{\prime}$ exist with $\beta\left(L_{1}^{\prime}\right)=\beta\left(L_{2}^{\prime}\right)=\beta_{0}$ such that

$$
L_{1}+L_{2}-\left(L_{t}+L_{t}^{*}\right)=L_{1}^{\prime}+L_{2}^{\prime}
$$

To establish this result, it clearly suffices to establish (8.1) directly for $\nu=2$. We therefore suppose $\beta\left(L_{0}\right)=2 \beta_{0}$, and proceed by induction on $N$ $=\sum n_{i}$. The case $N=1$ is vacuous. Suppose true for $N^{\prime}<N$.

Case 1. $Z^{2}(K, I)=0$. Then $\gamma\left(L_{0}\right)=2 \gamma$, since $\partial\left[\gamma\left(L_{0}\right)-2 \gamma\right]=0\left(K, \gamma\left(L_{0}\right)\right.$, and $\gamma$ being defined as above). It suffices for (8.1) to write $L_{0}=2 L_{\gamma}$, where $L_{\gamma}$ is defined as in the proof of (3.3).

Case 2. $Z^{2}(K, I) \neq 0$. Then since $K$ has no torsion, $z_{0}=\sum \epsilon_{i} t_{i}$ in $Z^{2}(K, I)$ exists such that all $\epsilon_{i}=0$ or \pm 1 and some $\epsilon_{i}=-1$ [1, pp. 277-283]. Let

$$
\gamma_{0}=\sum_{\epsilon_{i}=-1} t_{i} ; \quad \gamma^{\prime}=\gamma\left(L_{0}\right)-z_{0}-2 \gamma_{0}=\sum\left(n_{i}-\left|\epsilon_{i}\right|\right) t_{i} .
$$

Then

$$
\partial \gamma^{\prime}=\partial \gamma\left(L_{0}\right)-2 \partial \gamma_{0}=2\left(\zeta-\partial \gamma_{0}\right) .
$$

Suppose first that $\zeta=\partial \gamma_{0}$. Then for (8.1) it suffices to write

$$
L_{0}=\left(L_{0}-L_{\gamma_{0}}\right)+L_{\gamma_{0}} \text {. }
$$

Note that $L_{0}-L_{\gamma_{0}}$ is non-negative and so is a polyhedron, since all $n_{i} \geqq 1$ and

$$
L_{\gamma_{0}}=\sum_{\epsilon_{i}=-1} L_{t_{i}} \text {. }
$$

Suppose then that $\zeta \neq \partial \gamma_{0}$. Then $\zeta-\partial \gamma_{0}$ corresponds to a closed polygonal path $\beta_{1} \neq 0$ and, by (8.4), $2 \beta_{1}$ bounds $L_{\gamma^{\prime}}$. Now

$$
L_{\gamma^{\prime}}=\sum\left(n_{i}-\left|\epsilon_{i}\right|\right) L_{t_{i}} .
$$

Since $\sum\left(n_{i}-\left|\epsilon_{i}\right|\right)<N$, the induction hypothesis yields

$$
L_{\gamma^{\prime}}=L^{\prime}+L^{\prime \prime} \text {, }
$$

where $\beta\left(L^{\prime}\right)=\beta\left(L^{\prime \prime}\right)=\beta_{1}$. Then for (8.1) it suffices to note that

$$
L_{0}=\left(L^{\prime}+L_{z_{0}+\gamma_{0}}\right)+\left(L^{\prime \prime}+L_{\gamma_{0}}\right) \text {. }
$$


(8.5) is an immediate consequence of (8.3) as soon as it is observed that no $t_{i}^{*}$ is a face of any polyhedron in (8.5) and hence that no double triangle terms appear in that sum. This completes the proof of (8.1) for $\nu=2$, and so (as noted above) for arbitrary $\nu$.

REMARK. (8.1) is not true for $m>3$. For instance, a Klein bottle has a realization in $R^{4}$ which does not intersect itself. If we let $L_{0}$ denote a polyhedron realized in $R^{4}$ by such a bottle, then $\beta\left(L_{0}\right)=2 \beta$ where $\beta$ corresponds to the rim of the bottle. $L_{0}$ is not the sum of two polyhedra with $e$-boundary $\beta$.

Upon applying (8.1) at the appropriate point in the first paragraph of the proof of (7.2), and noting that for the case $\lambda_{0}=0$ it was shown in (7.2) that one can suppose all $\nu_{j}=1$ ( $m$ arbitrary), we get the desired extension:

(8.6) In case $m=3$, one can suppose in (7.2) that all $\nu_{j}=1$.

\section{BASIC $(\lambda)$; BASIC CLOSED}

9. Irreducible closed polyhedra. A closed polyhedron $L$ is termed irreducible if it has no decomposition $L=L_{1}+L_{2}$ into closed polyhedra $L_{1}, L_{2}$. Evidently:

(9.1) Every closed polyhedron is a sum of irreducible closed polyhedra and double triangles.

In particular, if $L$ is irreducible closed then $L$ has no double triangles. When the dimension $m$ is 3 , we have the following simple characterization of irreducible closed polyhedra:

(9.2) Suppose $m=3$. A necessary and sufficient condition that $L$ be irreducible closed is that $L$ have a realization in $R^{3}$ as a geometric polyhedron $P(L)$ separating $R^{3}$ into precisely two components of which it is the common boundary.

(9.2) follows from the fact that if $K$ is a euclidean 2-complex in $R^{3}$ generated by triangles $t_{1}, \cdots, t_{n}$ and $P=t_{1} \cup \cdots \cup t_{n}$, then the number of bounded components of $R^{3}-P$ is the number of independent elements of $Z^{2}(K, I)[1$, p. 380$]$.

10. Theorems (1.2) and (1.3.). The notions of basic $(\lambda)$ and basic closed as defined in $\$ 1$ are intimately connected with the notion of extreme point. For $\lambda \neq 0$, the $L$ basic $(\lambda)$ are, by definition, the extreme points of the set of all non-negative linear $L$ with $g$-boundary $\lambda(L)=\lambda$, while for $\lambda=0$ one has:

(10.1) $L_{0}$ is basic closed if and only if $\left[a\left(L_{0}\right)\right]^{-1} L_{0}$ is an extreme point of the set of all closed non-negative linear functionals $L$ of norm 1.

The importance here of the notion of extreme point lies in the following two principles, valid for sets of non-negative linear $L$ situated in a fixed compact subset of $R^{m}$ : First, every extreme point of the $w^{*}$ closed convex span of a set $\Gamma$ is in the $w^{*}$ closure of $\Gamma$; and second, the minimum of $\left(L, f_{0}\right)$ over a $w^{*}$ closed, convex set $\Gamma$ is attained at an extreme point of $\Gamma$ if it is attained at all in $\Gamma$. These principles are discussed further in the appendix.

Proof of Theorems (1.2) and (1.3). Let $\lambda \neq 0$ be admissible and $L_{0}$ basic ( $\lambda$ ). By Theorem (1.1), $L_{0}$ is the $w^{*}$ limit of a sequence of convex combina- 
tions $\sum k_{j} L_{j}$ of Dirichlet surfaces $L_{j}$ bounded by $\lambda$. Since the sequence converges $w^{*}$, its terms are situated in a fixed compact subset $A$ of $R^{m}$. By (A1) (see Appendix), $L_{0}$ is the $w^{*}$ limit of a sequence $\left(L_{n}\right)$ of Dirichlet surfaces situated in $A$ such that $\lambda\left(L_{n}\right)=\lambda$ for all $n$, which proves Theorem (1.2).

The proof of Theorem (1.3) is a little more involved. Let $L_{0}$ be basic closed; we may suppose $a\left(L_{0}\right)=1$. By Theorem (1.1), $L_{0}$ is the $w^{*}$ limit of a sequence $\sum k_{j} L_{j}$ of positive linear combinations of closed polyhedra $L_{j}$. Using (9.1) and approximating double triangles by flat tetrahedra, one may suppose that all $L_{j}$ are irreducible. As before, all $L_{j}$ are situated in some fixed compact set $A$ since the sequence converges $w^{*}$. Let $\Gamma$ denote the set of all $L$ of the form $\left[a\left(L_{1}\right)\right]^{-1} L_{1}$ where $L_{1}$ is an irreducible closed polyhedron situated in $A$. Now

$$
\eta=\sum k_{j} a\left(L_{j}\right)=\sum k_{j}\left(L_{j}, f_{a}\right) \rightarrow\left(L_{0}, f_{a}\right)=a\left(L_{0}\right)=1 ;
$$

hence, by replacing all $k_{j}$ by $\eta^{-1} k_{j}$, one can suppose all $\sum k_{j} a\left(L_{j}\right)=1$. It follows that $L_{0}$ is the $w^{*}$ limit of the convex sums of $\Gamma$ :

$$
\sum\left\{k_{j} a\left(L_{j}\right)\right\}\left\{\left[a\left(L_{j}\right)\right]^{-1} L_{j}\right\} .
$$

By (10.1) and (A1), $L_{0}$ is the $w^{*}$ limit of a sequence in $\Gamma$; thus, a sequence $\left(L_{n}\right)$ of irreducible closed polyhedra situated in $A$ exists such that

$$
L_{0}=w^{*} \lim _{n}\left[a\left(L_{n}\right)\right]^{-1} L_{n} .
$$

There are three cases:

Case 1. For a subsequence of $n, a\left(L_{n}\right) \rightarrow a_{0}>0$. Then $a_{0} L_{0}=w^{*} \lim _{n} L_{n}$.

Case 2: $a\left(L_{n}\right) \rightarrow 0$. Choose a sequence $\left(N_{n}\right)$ of positive integers such that $N_{n} a\left(L_{n}\right) \rightarrow 1$. For every $n=1,2, \cdots, N_{n} L_{n}$ is a closed polyhedron, and $L_{0}$ $=\lim _{n} N_{n} L_{n} . L_{0}$ can, in fact, be shown to be concentrated in this case.

Case 3: $a\left(L_{n}\right) \rightarrow \infty$. It is known that for $m=3$, every concentrated closed $L$ is the $w^{*}$ limit of a sequence of closed polyhedra $[18,(9.2)]$. We suppose $L_{0}$ not concentrated and derive a contradiction.

For arbitrary non-negative linear $L$, we denote by $s p t L$ the smallest set in which $L$ is situated. Since $L_{0}$ is not concentrated, spt $L_{0}$ contains two distinct points $x_{1}, x_{2}$. Let $\pi$ be a plane separating $x_{1}$ and $x_{2}$. Let

$$
L_{n}=L_{n}^{\prime}+L_{n}^{\prime \prime},
$$

where $L_{n}^{\prime}$ is situated on the side of $\pi$ containing $x_{1}$, and $L_{n}^{\prime \prime}$ on the side containing $x_{2}$. Since $\left[a\left(L_{n}\right)\right]^{-1} L_{n}^{\prime},\left[a\left(L_{n}\right)\right]^{-1} L_{n}^{\prime \prime}$ have bounded norms and are situated in a fixed bounded set, a subsequence of $n$ and $L^{\prime}, L^{\prime \prime}$ exist (by a well known selection process) such that

$$
L^{\prime}=w^{*} \lim _{n}\left[a\left(L_{n}\right)\right]^{-1} L_{n}^{\prime} ; \quad L^{\prime \prime}=w^{*} \lim _{n}\left[a\left(L_{n}\right)\right]^{-1} L_{n}^{\prime \prime} .
$$


By (9.2), each $L_{n}$ has a geometric realization $P\left(L_{n}\right)$ separating $R^{3}$ into exactly two components. $L_{n}^{\prime}$ and $L_{n}^{\prime \prime}$ may be closed up, for each $n=1,2, \cdots$, by adding polyhedra realized by the (suitably oriented) portion of $\pi$ interior to $P\left(L_{n}\right)$. Since these additions have area less than $\pi b^{2} / 4$ where $b$ is the diameter of $A$ and $a\left(L_{n}\right) \rightarrow \infty$, the additions contribute nothing to (10.2) in the limit. Thus $L^{\prime}$ and $L^{\prime \prime}$, being the $w^{*}$ limits of closed functionals, are closed. Spt $L^{\prime}$ contains $x_{1}$ and lies on the side of $\pi$ containing $x_{1}$; spt $L^{\prime \prime}$ lies on the opposite side of $\pi$ and contains $x_{2}$. Then $L_{0}=L^{\prime}+L^{\prime \prime}$, and $L^{\prime}=k L_{0}$ for no $k$, contradicting the assumption that $L_{0}$ is basic closed. This completes the proof of Theorem (1.3).

Remark. Theorem (1.2) is false for $m>3$. For example, let $L$ correspond as in $\S 8$ to a Klein bottle in $R^{4}$ which does not intersect itself and $\lambda$ to the rim of the bottle. Then $L / 2$ is basic $(\lambda)$; yet $L / 2$ can be shown not to be a $w^{*}$ limit of a sequence of Dirichlet surfaces with $g$-boundary $\lambda$. It is not known whether Theorem (1.3) is true for $m>3$.

11. Proof of Theorem (1.4). The necessity of (a) in (1.4) is a trivial consequence of the non-negativeness of the functionals $L$ involved and the definition of closed. We next establish that (a) implies (b). For, suppose (b) does not hold. Then there exists a sequence $\left(L_{n}\right)$ of closed polyhedra such that $L_{n}$ is situated in the $n^{-1}$ neighborhood of $A$ and

$$
\lim \sup _{n}\left[a\left(L_{n}\right)\right]^{-1}\left(L_{n}, f_{0}\right) \leqq 0 .
$$

Since the functionals $\left[a\left(L_{n}\right)\right]^{-1} L_{n}$ have bounded norms and $A$ is compact, a subsequence converges $w^{*}$ to $L_{1}$ situated in $A . L_{1}$ is closed, $a\left(L_{1}\right)=1$, and $\left(L_{1}, f_{0}\right) \leqq 0$. Hence

$$
\mu=\inf \left(L, f_{0}\right) \leqq 0,
$$

where the inf is taken over the set $\Gamma$ of all closed $L$ of norm 1 situated in $A$. By the Krein-Milman theorem, $\mu$ is attained at an extreme point of $\Gamma$, which by (10.1) implies that (a) does not hold. Thus (a) implies (b).

To complete the proof of Theorem (1.4), it suffices to show that (b) is sufficient. To this end, choose an open, convex set $\Omega_{1}$ such that $A \subset \Omega_{1} \subset \Omega$ and apply Lemma (5.1) to $f_{0}^{\prime}=f_{0}-\epsilon_{1} f_{a}$.

Theorem (1.4) has been stated in two equivalent forms. Condition (b) has the advantage of being stated directly in terms of the elementary notion of closed polyhedron. On the other hand, condition (a) is sometimes more convenient to apply (cf. (13.2) below).

\section{Applications}

12. Closed with 2-acyclic support. We again restrict attention to the case $m=3$. One thinks intuitively of a closed surface in $R^{3}$ as being situated in a number of 2-cycles. This is not necessarily the case for more general 
closed non-negative linear functionals $L$. In fact, a closed $L$ may be situated in a set $X$ which is acyclic in dimension 2 ; however, we shall show that such an $L$ degenerates (under certain restrictions on $X$ ) into a limit of sums of concentrated closed functionals.

For $X \subset R^{3}$ and $\epsilon>0$, let $X_{\epsilon}$ denote the $\epsilon$-neighborhood of $X$.

(12.1) Suppose $m=3$. Let $X$ be a compact set such that $R^{3}-X_{\epsilon}$ is connected for sufficiently small $\epsilon$, and such that given $x_{1}, x_{2}$ in $X$ there exists a plane $\pi$ separating $x_{1}$ and $x_{2}$ for which $|X \cap \pi|_{2}=0$. Then given a non-concentrated closed $L_{0}$ situated in $X$, there exist a plane $\pi$ and closed $L_{1}, L_{2}$ situated on opposite sides of $\pi$ (and not situated entirely in $\pi$ ) such that $L_{0}=L_{1}+L_{2}$.

REMARK. If, in particular, $X$ is a piecewise smooth 2-dimensional subset of $R^{3}$ and $R^{3}-X$ is connected, then the hypotheses of (12.1) hold.

Proof of (12.1). Let $\epsilon>0$ be small enough that $R^{3}-X_{\epsilon}$ is connected. Let $L_{0}$ be closed, nonconcentrated, and situated in $X$. By (1.1), $L_{0}$ is the $w^{*}$ limit of a sequence of convex combinations $\sum k_{j} L_{j}$ of closed polyhedra $L_{j}$.

We show first that all $L_{j}$ may be modified so as to be situated in $X_{\epsilon}$ in such a way that the sums $\sum k_{j} L_{j}$ still converge $w^{*}$ to $L_{0}$. To this end, let $\Omega$ be open with piecewise linear boundary and such that $X \subset \Omega \subset X_{\epsilon / 3}$. Using (9.1) and approximating double triangles by flat tetrahedra, one may suppose all $L_{j}$ irreducible closed, so that each $L_{j}$ has a geometric realization $P\left(L_{j}\right)$ as in (9.2). By trivial modifications, one can suppose no face of any $L_{j}$ parallel to a face of the boundary of $\Omega$. Let $L_{j}^{\prime}$ denote the portion of $L_{j}$ situated in $R^{3}-\Omega$. Let $f_{1}$ coincide with $f_{a}$ for $x$ in $R^{3}-\Omega$ and vanish for $x$ in $X$. Then

$$
\sum k_{j} a\left(L_{j}^{\prime}\right)=\sum k_{j}\left(L_{j}^{\prime}, f_{1}\right) \leqq \sum k_{j}\left(L_{j}, f_{1}\right) .
$$

The right side, and hence also the left side, of this expression tends to 0 since $\sum k_{j} L_{j}$ tends $w^{*}$ to $L_{0}$ situated in $X$. Let $a_{0}$ denote the area of the boundary $P$ of $\Omega$. If $a\left(L_{j}^{\prime}\right) \geqq \epsilon^{2} / 9$, replace $L_{j}^{\prime}$ by the portion of $P$ interior to $P\left(L_{j}\right)$ with orientation chosen to agree with that of $L_{j}$. This increases $a\left(L_{j}^{\prime}\right)$ by a factor of no more than $9 a_{0} \epsilon^{-2}$. Suppose $a\left(L_{j}^{\prime}\right)<\epsilon^{2} / 9$. Given $\delta>0$, let $\beta_{\delta}$ denote the closed polygonal path realized by the intersection of $P\left(L_{j}^{\prime}\right)$ with the boundary of the $\delta$-neighborhood $\Omega_{\delta}$ of $\Omega$. Letting $\rho\left(\beta_{\delta}\right)$ denote the length of $\beta_{\delta}$, it is elementary to verify that

$$
a\left(L_{j}^{\prime}\right) \geqq \int_{0}^{\infty} \rho\left(\beta_{\delta}\right) d \delta .
$$

It follows, in particular, that

$$
\rho\left(\beta_{\delta}\right) \leqq 3 a\left(L_{j}^{\prime}\right) \epsilon^{-1}<\epsilon / 3
$$

for at least one $\delta$ in $(0, \epsilon / 3)$. For this $\delta, \beta_{\delta}$ is situated in $X_{2 \epsilon / 3}$ and is of length at most $\epsilon / 3$. Its convex span is therefore contained in $X_{\epsilon}$. Then $\beta_{\delta}$ bounds a polyhedron situated in $X_{\epsilon}$ whose area approximates arbitrarily closely the 
inf for polyhedra bounded by $\beta_{\delta}$, and so by the isoperimetric inequality has area arbitrarily little more than $(1 / 4 \pi)\left[\rho\left(\beta_{\delta}\right)\right]^{2}$. But

$$
\frac{1}{4 \pi}\left[\rho\left(\beta_{\delta}\right)\right]^{2} \leqq \frac{9}{4 \pi}\left[a\left(L_{j}^{\prime}\right)\right]^{2} \epsilon^{-2}<\frac{a\left(L_{j}^{\prime}\right)}{4 \pi} .
$$

Replace the portion of $L_{j}^{\prime}$ exterior to $\Omega_{\delta}$ (if such exists) by this polyhedron.

These modifications increase the sums $\sum k_{j} a\left(L_{j}^{\prime}\right)$ by a factor of at most the fixed constant $\max \left[1 / 4 \pi, 9 a_{0} \epsilon^{-2}\right]$, so that these sums still converge to 0 . It follows that $\sum k_{j} L_{j}$ still tends $w^{*}$ to $L_{0}$.

By (9.1) one can again suppose the modified $L_{j}$ to be irreducible closed. Since $R^{3}-X_{\epsilon}$ is connected and each $P\left(L_{j}\right)$ is contained in $X_{\epsilon}$, the interior component of $R^{3}-P\left(L_{j}\right)$ is contained in $X_{\epsilon}$ for each $L_{j}$.

Choose a sequence $\left(\epsilon_{n}\right)$ tending to 0 . By the above remarks and the fact that the $w^{*}$ topology is metrizable in the set of non-negative linear $L$ situated in $X_{\epsilon_{1}}$ (assume $\epsilon_{1} \geqq \epsilon_{n}$ for all $n$ ), there exists a sequence of convex linear combinations $\sum_{j} k_{j n} L_{j n}$ tending $w^{*}$ to $L_{0}$ such that, for each $j$ and $n, L_{j n}$ is irreducible closed and the interior component of $P\left(L_{j n}\right)$ is contained in $X_{\epsilon_{n}}$. Let $x_{1}, x_{2}$ be distinct points of spt $L_{0}$, and $\pi$ a plane separating $x_{1}$ and $x_{2}$ such that $|X \cap \pi|_{2}=0$. Each $L_{j n}$ may be split into closed polyhedra by adding the (suitably oriented) portions of $\pi$ interior to $P\left(L_{j n}\right)$, which have area at most $\left|X_{\epsilon_{n}} \cap \pi\right|_{2}$ since the interior of $P\left(L_{j n}\right)$ is contained in $X_{\epsilon_{n}}$. Since

$$
\lim _{n}\left|X_{\epsilon_{n}} \cap \pi\right|_{2}=|X \cap \pi|_{2}=0
$$

and $\sum_{j} k_{j n}=1$ for all $n$, these added portions contribute nothing in the limit. Thus, proceeding as in the proof of Theorem (1.3), $L_{0}=L_{1}+L_{2}$ where $L_{1}$ and $L_{2}$ are closed and spt $L_{1}$ is on one side of $\pi$ and contains $x_{1}$ and spt $L_{2}$ is on the other side and contains $x_{2}$.

An immediate corollary of (12.1) is:

(12.2) If $L$ is basic closed and situated in a set $X$ satisfying the hypotheses of (12.1), then $L$ is concentrated.

Under slightly stronger restrictions on $X$, we prove:

(12.3) If $X$ is a compact set such that $R^{3}-X_{\epsilon}$ is connected for sufficiently small $\epsilon$, and if parallel to each coordinate plane a nondenumerable dense set of planes $\pi$ exists for which $|X \cap \pi|_{2}=0$, then every closed $L$ situated in $X$ is representable as a Bochner integral (in the McShane distance):

$$
L=\int_{X} L_{x} d \omega(x)
$$

where $\omega$ is a Borel measure vanishing outside $X$ and each $L_{x}$ is closed and concentrated at $x$.

Proof. From known theorems, it follows that every non-negative linear 
$L$ situated in $X$ has the form of a Bochner integral (12.4) (in the McShane distance), where $\omega$ is a Borel measure vanishing on $R^{3}-X$ and $L_{x}$ is concentrated at $x$. Except for a set of $\omega$ measure $0, L_{x}$ is the derivative of the integral (12.4) with respect to a network of intervals $D$ bounded by planes $\pi$ for which $|X \cap \pi|_{2}=\omega(X \cap \pi)=0$. It follows from (12.1) that $\int_{D} L_{x} d \omega$ is closed for each such $D$; thus, $L_{x}=\lim _{D \rightarrow x}[\omega(D)]^{-1} \int_{D} L_{x} d \omega$ is closed except for a set of $\omega$ measure 0 , on which $L_{x}$ may be defined arbitrarily. This proves (12.3).

We observe next that if $L$ is a non-negative linear functional concentrated at a point $x_{0}, L$ is closed if and only if $L$ has generalized track 0 . This follows at once from the definitions, the linearity in $J$ of exact integrands, and the remark that if $f(x, J)$ is an arbitrary integrand which is linear in $J$, then $\phi(J)=f\left(x_{0}, J\right)$ is exact. From this it follows at once that each integral (12.4) in which the $L_{x}$ are closed and concentrated has generalized track 0 . The converse is also true. To establish it write $L$ with generalized track 0 in the form (12.4), where each $L_{x}$ is concentrated at $x$ and except for a set of $\omega$ measure 0 is the derivative of (12.4). Let $\pi$ be a plane such that $\omega(\pi)=0$, and let $L_{1}, L_{2}$ denote the portions of $L$ situated on opposite sides of $\pi$. Let $f(x, J)$ in $E^{m}$ be linear in $J$, and let $\left(f_{n}\right)$ be a sequence of integrands in $E^{m}$ linear in $J$ such that $f_{n}$ coincides with $f$ on the side of $\pi$ in which $L_{1}$ is situated, vanishes at all points in the other at distance more than $n^{-1}$ of $\pi$, and has the same bounds as $f$. Then

$$
\left(L_{1}, f\right)=\lim _{n}\left(L, f_{n}\right)=0 ;
$$

i.e., $L_{1}$ has generalized track 0 . Similarly, $L_{2}$ has generalized track 0 . Proceeding as in the proof of (12.3), we find that, except for a set of $\omega$ measure $0, L_{x}$ has generalized track 0 , and so being concentrated is closed. We have:

(12.5) A necessary and sufficient condition that $L$ have generalized track 0 is that $L$ be of the form (12.4), where $\omega$ is a Borel measure on $R^{m}$ vanishing outside some compact set $X$ and each $L_{x}$ is closed and concentrated at $x$.

13. An existence theorem for an ordinary minimum problem. Consider the following:

(13.1) Minimum problem: Let $A \subset R^{3}$ be compact and convex. Find the minimum $\mu=\inf \left(L, f_{0}\right)$ in the set $\Gamma$ of all Dirichlet surfaces $L$ situated in $A$ which have Dirichlet representations on the unit square $U$ reducing on the perimeter of $U$ to representations of a given simple closed Fréchet curve $C$.

According to Cesari [3] (for similar results arrived at independently of [3] and each other, see Danskin [6] and Sigalov [13]), the problem (13.1) has a solution in $\Gamma$, provided $\Gamma$ is nonvoid and:

(1) $f_{0}$ is in $E^{3}$;

(2) $f_{0}$ is positive semi-regular, i.e., $\psi(J)=f_{0}\left(x_{0}, J\right)$ is convex for each $x_{0}$ in $A ;$ and

(3) $f_{0}$ is positive definite, i.e., $f_{0}(x, J)>0$ for $x$ in $A$ and $J \neq 0$. 
The purpose of this section is to show that condition (3) may be weakened, as follows:

(13.2) Existence TheOREM. The minimum problem (13.1) has a solution in $\Gamma$ if $C$ is rectifiable and contained in $A$, and if (1), (2), and any one of the following hold:

(3a) $\left(L, f_{0}\right)>0$ for every basic closed $L$ situated in $A$;

(3b) There exists an open set $\Omega$ containing $A$ and $\epsilon_{1}>0$ such that $\left(L, f_{0}\right)$ $\geqq \epsilon_{1} a(L)$ for every closed polyhedron $L$ situated in $\Omega$; or

(3c) (i) $f_{0}(x, J) \geqq 0$, whenever $x$ is in $A$;

(ii) $f_{0}(x, J)+f_{0}(x,-J)>0$, for $x$ in $A$ and $J \neq 0$;

(iii) The zeros $\left(^{6}\right)$ of $f_{0}$ are contained in a closed subset $X$ of $A$ satisfying the hypotheses of $(12.1)\left({ }^{7}\right)$.

Proof. Since $C$ is rectifiable and contained in $A, \Gamma$ is nonvoid. Suppose (3a) or (3b) holds. By Theorem (1.4), an exact integrand $\phi$ and an $\epsilon>0$ exist such that $f_{0}(x, J)-\phi(x, J) \geqq \epsilon|J|$ for all $x$ in $A$ and all $J$. The integrand $f_{0}-\phi$ is thus positive definite. Since $\phi$ is linear in $J$ by (4.4) and $f_{0}$ is semiregular, $f_{0}-\phi$ is semi-regular. By the existence theorem cited above for positive definite, semi-regular integrands, an $L_{0}$ in $\Gamma$ exists such that $\left(L_{0}, f_{0}-\phi\right)$ $\leqq\left(L, f_{0}-\phi\right)$ for all $L$ in $\Gamma$. By $(6.3), \lambda\left(L_{0}\right)=\lambda(L)$ for all $L$ in $\Gamma$. Then, for all $L$ in $\Gamma$,

$$
\begin{aligned}
\left(L_{0}, f_{0}\right) & =\left(L_{0}, f_{0}-\phi\right)+\left(L_{0}, \phi\right)=\left(L_{0}, f_{0}-\phi\right)+\left(\lambda\left(L_{0}\right), \phi\right) \\
& \leqq\left(L, f_{0}-\phi\right)+(\lambda(L), \phi)=\left(L, f_{0}-\phi\right)+(L, \phi)=\left(L, f_{0}\right) ;
\end{aligned}
$$

i.e., $L_{0}$ is a solution for the minimum problem (13.1).

Suppose (3c) holds. Let $L_{1}$ be basic closed and situated in $A$. If $L_{1}$ is not situated in $X$, then $\left(L_{1}, f_{0}\right)>0$ since $f_{0}$ is non-negative and strictly positive for $x$ in $A-X$ and $J \neq 0$. If, on the other hand, $L_{1}$ is situated in $X$ then, by (12.2), $L_{1}$ is concentrated at a point $x_{0}$ of $X$. Let $\psi(J)=f_{0}\left(x_{0}, J\right)$. Then $\psi(k J)$ $=k \psi(J)$ for $k \geqq 0$, and $\psi$ is convex since $f_{0}$ is semi-regular. Since the domain $S^{3}$ of $\psi$ is a linear space (every skew symmetric $3 \times 3$ matrix $J$ being of rank 0 or 2), it follows from these properties and (3c) (ii) that a linear function $\phi(J)$ exists such that $\phi(J)<\psi(J)$ for $J \neq 0$. As noted in the remarks following (4.5), $\phi$ is an exact integrand. Then since $L_{1}$ is closed, non-negative, and not 0 :

$$
0=\left(L_{1}, \phi\right)<\left(L_{1}, \psi\right)=\left(L_{1}, f_{0}\right) .
$$

Thus (3c) implies (3a). This completes the proof of (13.2).

14. Generalized minimum problems. Minimum problems of the type of (13.1) may cease to have solutions identifiable as surfaces in the ordinary

( $\left.{ }^{6}\right)$ A point $x_{0}$ in $R^{3}$ is called a zero of $f_{0}$ if $f_{0}\left(x_{0}, J\right)=0$ for at least one $J \neq 0$.

${ }^{(7)}$ This theorem remains true if $C$ merely occupies 2-dimensional Hausdorff measure 0 and $\Gamma$ is nonvoid (see footnote 5 ). 
sense if the condition of semi-regularity of the integrand $f_{0}$ is dropped. To establish a general principle of minimum applicable to nonregular as well as regular problems, we therefore define to be a generalized solution (or, generalized surface solution) the limit of a $w^{*}$ convergent minimizing sequence of Dirichlet surfaces. The existence of a generalized solution is insured as soon as a minimizing sequence with bounded norms (=Lebesgue areas) and situated in a fixed bounded subset of $R^{m}$ exists [18, p. 64, Principle of Minimum ]. We note further that by (A1) and (A4) below, the existence of a generalized solution to the minimum problem $\mu=\inf \left(L, f_{0}\right)$ over a set $\Gamma$ of Dirichlet surfaces situated in a bounded subset of $R^{m}$ is equivalent to the existence of a solution in the $w^{*}$ closed convex span $c(\Gamma)$ of $\Gamma$. This is in turn equivalent to the existence of a hyperplane of support to $c(\Gamma)$ of the form $\left(L, f_{0}\right)=\mu$.

\section{ApPENDIX}

In this appendix we prove two results ((A1) and (A4)) on extreme points, which we have referred to above. (A4) is a minor extension of the well known Krein-Milman theorem [8]. For completeness we give a direct proof of (A1) rather than attempt to deduce it from the bounded case, which is known (cf. Milman [11]), but apparently much less well known than the KreinMilman theorem. The proof of (A1) given applies with only trivial modifications in establishing Milman's result, namely, that the extreme points of the $w^{*}$ closed convex span of an arbitrary bounded subset $\Gamma$ of the conjugate of a Banach space lie in the $w^{*}$ closure of $\Gamma$.

(A1) Let $A \subset R^{m}$ be compact. Then every extreme point of the w* closed convex span $c(\Gamma)$ of a set $\Gamma$ of non-negative linear functionals $L$ situated in $A$ is the w* limit of a sequence in $\Gamma$.

Proof. Suppose (A1) false. Then an extreme point $L_{0}$ of $c(\Gamma)$, and a $w^{*}$ neighborhood

$$
N=N\left(L_{0} ; \epsilon ; f_{1}, \cdots, f_{\nu}\right)=\left[L||\left(L, f_{j}\right)-\left(L_{0}, f_{j}\right) \mid<\epsilon, j=1, \cdots, \nu\right]
$$

exist such that $N \cap \Gamma$ is void. By adjoining $f_{a}$ to the set $f_{1}, \cdots, f_{\nu}$ if necessary, one can suppose that $f_{1}=f_{a}$. Let $N_{1}=N\left(L_{0} ; \epsilon / 2 ; f_{1}, \cdots, f_{v}\right)$. Since $L_{0}$ is in $c(\Gamma), L_{0}$ is the $w^{*}$ limit of a directed sequence of convex combinations

$$
L_{\alpha}=\sum_{i=1}^{i_{\alpha}} k_{i \alpha} L_{i \alpha}
$$

of elements $L_{i \alpha}$ of $\Gamma$. Since $L_{0}=w^{*} \lim _{\alpha} L_{\alpha}$, all $L_{\alpha}$ are in $N_{1}$ for $\alpha>\alpha_{0}$. Suppose that

$$
\left|\left(L_{i \alpha}, f_{j}\right)-\left(L_{\alpha}, f_{j}\right)\right|<\epsilon / 2
$$

for all $j=1, \cdots, \nu$. Then a straightforward verification shows that $L_{i \alpha}$ would be in $N$ for $\alpha>\alpha_{0}$, which is not possible since all $L_{i \alpha}$ are in $\Gamma$ and $\Gamma \cap N$ 
is void. Hence each $i=1,2, \cdots, i_{\alpha}$ for $\alpha>\alpha_{0}$ is in at least one of the sets

$$
\begin{aligned}
& Q^{+}(j, \alpha)=\left[i \mid\left(L_{i \alpha}, f_{j}\right)-\left(L_{\alpha}, f_{j}\right) \geqq \epsilon / 2\right] ; \\
& Q^{-}(j, \alpha)=\left[i \mid\left(L_{i \alpha}, f_{j}\right)-\left(L_{\alpha}, f_{j}\right) \leqq-\epsilon / 2\right] .
\end{aligned}
$$

Let

$$
k^{ \pm}(j, \alpha)=\sum_{i \in Q_{ \pm}(j, \alpha)} k_{i \alpha}
$$

Then for $\alpha>\alpha_{0}$,

$$
\sum_{j=1}^{\nu}\left[k^{+}(j, \alpha)+k^{-}(j, \alpha)\right] \geqq \sum_{i=1}^{i_{\alpha}} k_{i \alpha}=1
$$

Hence at least one $k^{ \pm}(j, \alpha)=k_{\alpha} \geqq 1 / 2 \nu$. Let $Q_{\alpha}$ denote the corresponding $Q^{ \pm}(j, \alpha)$, and

$$
L_{\alpha}^{\prime}=k_{\alpha}^{-1} \sum_{i \in Q_{\alpha}} k_{i \alpha} L_{i \alpha} .
$$

Since $f_{1}=f_{a}$ and all $L$ in question are non-negative,

$$
a\left(L_{\alpha}\right)=\left(L_{\alpha}, f_{1}\right)<\left(L_{0}, f_{1}\right)+\epsilon / 2=a\left(L_{0}\right)+\epsilon / 2
$$

for $\alpha>\alpha_{0}$. It follows that $a\left(L_{\alpha}^{\prime}\right) \leqq k_{\alpha}^{-1} a\left(L_{\alpha}\right) \leqq 2 \nu a\left(L_{\alpha}\right)<2 \nu a\left(L_{0}\right)+\nu \epsilon$. Since the set of all $L$ situated in $A$ with norms bounded by $2 \nu a\left(L_{0}\right)+\nu \epsilon$ is $w^{*}$ compact and $1 / 2 \nu \leqq k_{\alpha} \leqq 1$ for $\alpha>\alpha_{0}$, subdirected sequences $\left(k_{\beta}\right),\left(L_{\beta}^{\prime}\right)$ of $\left(k_{\alpha}\right),\left(L_{\alpha}^{\prime}\right)$ and $k, L^{\prime}$ exist such that $k=\lim _{\beta} k_{\beta}$ and $L^{\prime}=w^{*} \lim _{\beta} L_{\beta}^{\prime}$.

Now for each $\alpha>\alpha_{0}$,

$$
k_{\alpha}\left|\left(L_{\alpha}^{\prime}, f_{j}\right)-\left(L_{\alpha}, f_{j}\right)\right|=\left|\sum_{i \in Q_{\alpha}} k_{i \alpha}\left[\left(L_{i \alpha}, f_{j}\right)-\left(L_{\alpha}, f_{j}\right)\right]\right| \geqq k_{\alpha} \frac{\epsilon}{2} \geqq \frac{\epsilon}{4 \nu}
$$

for at least one $j$ by the choice of $Q_{\alpha}$. Since there are only a finite number of $f_{j}$, passage to the limit gives

$$
k\left|\left(L^{\prime}, f_{j}\right)-\left(L_{0}, f_{j}\right)\right| \geqq \epsilon / 4 \nu
$$

for at least one $j$.

If $k=1$, then $L^{\prime}=L_{0}$, contrary to (A3). Hence $k<1$. Now all $L_{\beta}^{\prime}$ are in $c(\Gamma)$ by their definition; hence $L^{\prime}$ is in $c(\Gamma)$ since $c(\Gamma)$ is $w^{*}$ closed. Since $k_{\beta} \rightarrow k$ $<1$,

$$
L_{\beta}^{\prime \prime}=\left(1-k_{\beta}\right)^{-1}\left(L_{\beta}-k_{\beta} L_{\beta}^{\prime}\right)
$$

converges $w^{*}$ to $L^{\prime \prime}=(1-k)^{-1}\left(L_{0}-k L^{\prime}\right)$ and $L_{0}=k L^{\prime}+(1-k) L^{\prime \prime}$. All $L_{\beta}^{\prime \prime}$ are in $c(\Gamma)$; hence, $L^{\prime \prime}$ is in $c(\Gamma)$. Since $L_{0}$ is an extreme point of $c(\Gamma), L_{0}=L^{\prime}=L^{\prime \prime}$, contradicting (A3). Thus every extreme point $L_{0}$ of $c(\Gamma)$ is a $w^{*}$ limit point of $\Gamma$. Since the $w^{*}$ topology is metrizable by the McShane distance in the set 
of non-negative linear functionals situated in $A$, each such extreme point is the limit of a sequence in $\Gamma$.

(A4) Let $A \subset R^{m}$ be compact and $f_{0}$ in $E^{m, A}$. Then the minimum $\mu$ $=\inf \left(L, f_{0}\right)$ over a $w^{*}$ closed convex set $\Gamma$ of non-negative linear functionals $L$ situated in $A$ is attained at an extreme point of $\Gamma$ if it is attained at all in $\Gamma$.

Proof. The set $\Gamma_{1}$ of $L$ in $\Gamma$ for which $\left(L, f_{0}\right)=\mu$ is $w^{*}$ closed, convex, and by hypothesis nonempty. Since $a(L)=\left(L, f_{a}\right)$ for $L$ non-negative, an $L_{1}$ in $\Gamma_{1}$ of smallest norm exists. The set $\Gamma_{2}$ of $L$ in $\Gamma$ such that $a(L)=a\left(L_{1}\right)$ and $\left(L, f_{0}\right)=\mu$ is $w^{*}$ compact and convex. By the Krein-Milman theorem [8], an extreme point $L_{0}$ of $\Gamma_{2}$ exists. It follows easily from the linearity of $a(L)$ and $\left(L, f_{0}\right)$ that $L_{0}$ is also an extreme point of $\Gamma$, which proves (A4).

\section{REFERENCES}

1. P. Alexandroff and H. Hopf, Topologie, Berlin, 1935.

2. S. Banach, Théorie des opérations linéaires, Warsaw, 1932.

3. L. Cesari, An existence theorem in the calculus of variations for integrals on parametric surfaces, Amer. J. Math. vol. 74 (1952) pp. 265-295.

4. W. Damköhler, Über indefinite Variationsprobleme, Math. Ann. vol. 110 (1935) pp. 230283.

5. W. Damköhler and E. Hopf, Über einige Eigenschaften von Kurvenintegralen und über die Aequivalenz von indefiniten mit definiten Variationsproblemen, Math. Ann. vol. 120 (1947-49) pp. 12-20.

6. J. M. Danskin, On the existence of minimizing surfaces in parametric double integral problems in the calculus of variations, Rivista Mat. Parma vol. 3 (1952) pp. 43-63.

7. W. V. D. Hodge, The theory and applications of harmonic integrals, Cambridge, 1941.

8. M. Krein and D. Milman, On extreme points of regularly convex sets, Studia Math. vol. 9 (1940) pp. 133-138. 1942.

9. S. Lefschetz, Algebraic topology, Amer. Math. Soc. Colloquium Publications, vol. 27,

10. E. J. McShane, Generalized curves, Duke Math. J. vol. 6 (1940) pp. 513-536.

11. D. Milman, Characteristics of extremal points of regularly convex sets, Doklady Akad. Nauk SSSR. N.S. vol. 57 (1947) pp. 119-122.

12. S. Saks, Theory of the integral, Warsaw, 1937.

13. A. G. Sigalov, Two dimensional problems in the calculus of variations, Uspehi Mat. Nauk N.S. vol. 6 (1951) pp. 16-101.

14. L. C. Young, Generalized curves and the existence of an attained absolute minimum in the calculus of variations, Comptes Rendus de la Société des Sciences et des Lettres de Varsovie, Classe III, vol. 30 (1937) pp. 212-234.

15. - Generalized surfaces in the calculus of variations, Ann. of Math. vol. 43 (1942) pp. 84-103.

16. - Generalized surfaces in the calculus of variations. II, Ann. of Math. vol. 43 (1942) pp. 530-544.

17. - Some applications of the Dirichlet integral to the theory of surfaces, Trans. Amer. Math. Soc. vol. 64 (1948) pp. 317-335.

18. - Surfaces paramétriques gênêralisées, Bull. Soc. Math. France vol. 79 (1951) pp. 59-85.

UNIVERSITY OF WISCONSIN, MADISON, Wis. 